\title{
Model-Independent Comparison Between Factorization Algebras and Algebraic Quantum Field Theory on Lorentzian Manifolds
}

\author{
Marco Benini $^{1,2}$ (D), Marco Perin ${ }^{3}$ (D), Alexander Schenkel ${ }^{3}$ (iD \\ 1 Fachbereich Mathematik, Universität Hamburg, Bundesstr. 55, 20146 Hamburg, Germany. \\ E-mail: benini@dima.unige.it \\ 2 Dipartimento di Matematica, Università di Genova, Via Dodecaneso 35, 16146 Genoa, Italy \\ 3 School of Mathematical Sciences, University of Nottingham, University Park, Nottingham NG7 2RD, UK. \\ E-mail: marco.perin@nottingham.ac.uk; alexander.schenkel@nottingham.ac.uk
}

Published online: 5 September 2019 - (C) The Author(s) 2019

\begin{abstract}
This paper investigates the relationship between algebraic quantum field theories and factorization algebras on globally hyperbolic Lorentzian manifolds. Functorial constructions that map between these two types of theories in both directions are developed under certain natural hypotheses, including suitable variants of the local constancy and descent axioms. The main result is an equivalence theorem between (Cauchy constant and additive) algebraic quantum field theories and (Cauchy constant, additive and time-orderable) prefactorization algebras. A concept of $*$-involution for the latter class of prefactorization algebras is introduced via transfer. This involves Cauchy constancy explicitly and does not extend to generic (time-orderable) prefactorization algebras.
\end{abstract}

\section{Contents}

1. Introduction and Summary . . . . . . . . . . . . . . . . . . . . . . 972

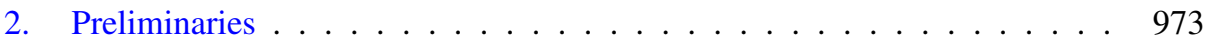

2.1 Lorentzian geometry . . . . . . . . . . . . . . . . . . . 973

2.2 Factorization algebras . . . . . . . . . . . . . . . . . . 975

2.3 Algebraic quantum field theories . . . . . . . . . . . . . . . . . . . . . 978

3. From PFA to AQFT . . . . . . . . . . . . . . . . . . . . . . . 979

3.1 Object-wise algebra structure . . . . . . . . . . . . . . . . . . . . . . . . . . . . . 979

3.2 Naturality of algebra structures . . . . . . . . . . . . . . . . . . . . . . . . . . 981

3.3 Algebraic quantum field theory axioms . . . . . . . . . . . . . . . . . . 983

4. From AQFT to PFA . . . . . . . . . . . . . . . . . . . . . . . . . . . . . . . . . . . . . . . . . . . . . .

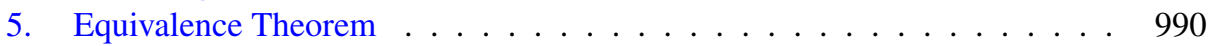

5.1 Main result . . . . . . . . . . . . . . . . . . . . . . . . . . . . . . . . . . . . . . .

5.2 Transfer of $*$-involutions . . . . . . . . . . . . . . . . . 993

5.3 Example: the free Klein-Gordon field . . . . . . . . . . . . . . . . . 994 


\section{Introduction and Summary}

Factorization algebras and algebraic quantum field theory are two mathematical frameworks to axiomatize the algebraic structure of observables in a quantum field theory. While from a superficial point of view these two approaches look similar, there are subtle differences. A prefactorization algebra $\mathfrak{F}$ assigns to each spacetime $M$ a vector space $\mathfrak{F}(M)$ of observables and to each tuple $f=\left(f_{1}: M_{1} \rightarrow N, \ldots, f_{n}: M_{n} \rightarrow N\right)$ of pairwise disjoint spacetime embeddings a factorization product $\mathfrak{F}(f): \bigotimes_{i=1}^{n} \mathfrak{F}\left(M_{i}\right) \rightarrow$ $\mathfrak{F}(N)$ satisfying suitable properties, cf. [CG17] and Sect. 2.2. On the other hand, an algebraic quantum field theory $\mathfrak{A}$ assigns to each spacetime $M$ an associative and unital *-algebra $\mathfrak{A}(M)$ of observables and to each spacetime embedding $f: M \rightarrow N$ a $*$-algebra morphism $\mathfrak{A}(f): \mathfrak{A}(M) \rightarrow \mathfrak{A}(N)$ such that suitable axioms hold true, cf. [BFV03,FV12,BDFY15, BSW17] and Sect. 2.3. The main differences are that, in contrast to an algebraic quantum field theory $\mathfrak{A}$, a prefactorization algebra $\mathfrak{F}$ does not in general come endowed with (1) a multiplication of observables in $\mathfrak{F}(M)$, i.e. on the same spacetime $M$, because $\left(\operatorname{id}_{M}: M \rightarrow M, \operatorname{id}_{M}: M \rightarrow M\right)$ is not a pair of disjoint spacetime embeddings, and (2) a concept of $*$-involution on observables in $\mathfrak{F}(M)$.

In this paper we shall develop functorial constructions (cf. Theorems 3.11 and 4.7) that allow us to relate prefactorization algebras and algebraic quantum field theories, provided that we assume certain natural hypotheses on both sides. We shall focus on the case where spacetimes are described by oriented and time-oriented globally hyperbolic Lorentzian manifolds, i.e. on the case of relativistic quantum field theory, and disregard until Sect. 5.2 the $*$-involutions on algebraic quantum field theories because prefactorization algebras are usually considered without a concept of $*$-involution. Our main result is an equivalence theorem between (Cauchy constant and additive) algebraic quantum field theories and (Cauchy constant, additive and time-orderable) prefactorization algebras, cf. Theorem 5.1. Our equivalence theorem is considerably more general than the earlier comparison result by Gwilliam and Rejzner [GR17]: (1) We work in a model-independent setup, supplemented by natural additional hypotheses such as Cauchy constancy, additivity and time-orderability, while [GR17] only studies linear quantum field theories, such as e.g. the free Klein-Gordon field. (2) We investigate in detail uniqueness, associativity, naturality and Einstein causality of the multiplications $\mu_{M}: \mathfrak{F}(M) \otimes \mathfrak{F}(M) \rightarrow \mathfrak{F}(M)$ determined by a Cauchy constant additive prefactorization algebra $\mathfrak{F}$, which requires rather sophisticated arguments from Lorentzian geometry. These questions were not addressed in [GR17]. (3) Our equivalence theorem admits an interpretation in terms of operad theory (cf. Remark 5.2), which provides a suitable starting point for generalizations to higher categorical quantum field theories [CG17, BSS15, BS17, BSW19b,BS19] such as gauge theories. (The present paper does not study this generalization and will focus on the case of 1-categorical quantum field theories.) We would like to state very clearly that our results prove an equivalence theorem between certain categories of prefactorization algebras and algebraic quantum field theories, hence they do not make any statements about the relationship between explicit construction methods for examples. We refer to [GR17] for a concrete comparison between BV quantization [CG17] and perturbative canonical quantization [FR13, Rej16].

Let us now explain in more detail our constructions and results while outlining the content of the present paper: in Sect. 2 we recall the necessary preliminaries from Lorentzian geometry, factorization algebras and algebraic quantum field theory. All prefactorization algebras and algebraic quantum field theories will be defined on the usual category Loc of oriented and time-oriented globally hyperbolic Lorentzian manifolds. We introduce an additivity axiom for both prefactorization algebras and algebraic quantum field theories, 
which roughly speaking demands that the observables in a spacetime $M$ are generated by the observables in the relatively compact and causally convex open subsets $U \subseteq M$. It is shown that factorization algebras, i.e. prefactorization algebras satisfying Weiss descent, are in particular additive prefactorization algebras. We also introduce a Cauchy constancy (or time-slice) axiom for both kinds of theories, which formalizes a concept of time evolution in a globally hyperbolic Lorentzian manifold. In Sect. 3 we construct a functor $\mathbb{A}: \mathbf{P F A}^{\text {add, } c} \rightarrow \mathbf{A Q F T}{ }^{\text {add }, c}$ that assigns a Cauchy constant additive algebraic quantum field theory $\mathbb{A}[\mathfrak{F}]$ to each Cauchy constant additive prefactorization algebra $\mathfrak{F}$, see Theorem 3.11 for the main result. The crucial step is to define canonical multiplications $\mu_{M}: \mathfrak{F}(M) \otimes \mathfrak{F}(M) \rightarrow \mathfrak{F}(M)$ for such $\mathfrak{F}$ (cf. (3.1)), which is done by using Cauchy constancy. Proving naturality and Einstein causality of these multiplications requires the additivity axiom, cf. Propositions 3.7 and 3.10. In Sect. 4 we construct a functor $\mathbb{F}:$ AQFT $\rightarrow$ tPFA that assigns a time-orderable prefactorization algebra $\mathbb{F}[\mathfrak{A}]$ to each algebraic quantum field theory $\mathfrak{A}$, see Theorem 4.7 for the main result. The difference between time-orderable and ordinary prefactorization algebras on Loc is that the former just encode factorization products $\mathfrak{F}(f): \bigotimes_{i=1}^{n} \mathfrak{F}\left(M_{i}\right) \rightarrow N$ for tuples of pairwise disjoint morphisms $f$ that are in a suitable sense time-orderable, see Definition 4.1. There is a natural forgetful functor PFA $\rightarrow$ tPFA from ordinary to time-orderable prefactorization algebras, which is however not full, see Remarks 4.2 and 4.5. Our results suggest that the concept of time-orderable prefactorization algebras from Sect. 4 is better suited to the category of Lorentzian spacetimes Loc than the more naive concept from Sect. 2.2 that allows also for factorization products for non-time-orderable tuples of pairwise disjoint morphisms. In Sect. 5 we explain that the construction $\mathbb{A}: \mathbf{P F A}^{\text {add }, c} \rightarrow \mathbf{A Q F T}^{\text {add }, c}$ from Sect. 3 factors through the forgetful functor $\mathbf{P F A}{ }^{\text {add }, c} \rightarrow$ tPFA $^{\text {add }, c}$, thereby defining a functor $\mathbb{A}:$ tPFA $^{\text {add }, c} \rightarrow$ AQFT $^{\text {add, } c}$ that assigns a Cauchy constant additive algebraic quantum field theory to each Cauchy constant additive time-orderable prefactorization algebra. Our main Equivalence Theorem 5.1 proves that this functor admits an inverse that is given by the restriction $\mathbb{F}: \mathbf{A Q F T}^{\text {add }, c} \rightarrow \mathbf{t P F A}^{\text {add }, c}$ of the functor from Sect. 4 to Cauchy constant and additive theories. Hence, Cauchy constant additive algebraic quantum field theories are naturally identified with Cauchy constant additive time-orderable prefactorization algebras. In Sect. 5.2, we use our main Equivalence Theorem 5.1 to transfer $*$-involutions from algebraic quantum field theories to Cauchy constant additive time-orderable prefactorization algebras. By construction, we obtain an equivalence $* \mathbf{A Q F T} \mathbf{T}^{\text {add }, c} \simeq * \mathbf{t P F A}^{\text {add, } c}$ between theories with $*$-involutions. We show that the transferred concept of $*$-involutions for Cauchy constant additive timeorderable prefactorization algebras involves Cauchy constancy explicitly, hence it does not extend to generic time-orderable prefactorization algebras in tPFA. In Sect. 5.3, we apply our general results to the simple example given by the free Klein-Gordon field $\mathfrak{A}_{\mathrm{KG}} \in \mathbf{A Q F T}{ }^{\text {add }, c}$. We observe as in [GR17] that the corresponding time-orderable prefactorization algebra $\mathfrak{F}_{\mathrm{KG}} \in \mathbf{t P F A}^{\text {add, } c}$ describes the time-ordered products from perturbative algebraic quantum field theory, cf. [FR13, Rej16].

\section{Preliminaries}

2.1. Lorentzian geometry. In order to fix our notations, we shall briefly recall some basic definitions and properties of Lorentzian manifolds. We refer to [BGP07] for a concise introduction.

A Lorentzian manifold is a manifold $M$ together with a metric $g$ of signature $(-+$ $\cdots+$ ). A non-zero tangent vector $0 \neq v \in T_{x} M$ at a point $x \in M$ is called time-like 
if $g(v, v)<0$, light-like if $g(v, v)=0$ and space-like if $g(v, v)>0$. It is called causal if it is either time-like or light-like, i.e. $g(v, v) \leq 0$. A curve $\gamma: I \rightarrow M$, where $I \subseteq \mathbb{R}$ is an open interval, is called time-like/light-like/space-like/causal if all its tangent vectors $\dot{\gamma}$ are time-like/light-like/space-like/causal. A Lorentzian manifold is called time-orientable if there exists a vector field $\mathfrak{t} \in \Gamma^{\infty}(T M)$ that is everywhere time-like. Such $\mathfrak{t}$ determines a time-orientation.

In what follows we always consider time-oriented Lorentzian manifolds, denoted collectively by symbols like $M$, suppressing the metric $g$ and time-orientation $t$ from our notation. A time-like or causal curve $\gamma: I \rightarrow M$ is called future directed if $g(\mathfrak{t}, \dot{\gamma})<0$ and past directed if $g(\mathfrak{t}, \dot{\gamma})>0$. The chronological future/past of a point $x \in M$ is the subset $I_{M}^{ \pm}(x) \subseteq M$ of all points that can be reached from $x$ by future/past directed time-like curves. The causal future/past of a point $x \in M$ is the subset $J_{M}^{ \pm}(x) \subseteq M$ of all points that can be reached from $x$ by future/past directed causal curves and $x$ itself. Given any subset $S \subseteq M$, we define $I_{M}^{ \pm}(S):=\bigcup_{x \in S} I_{M}^{ \pm}(x)$ and $J_{M}^{ \pm}(S):=\bigcup_{x \in S} J_{M}^{ \pm}(x)$.

Definition 2.1. Let $M$ be a time-oriented Lorentzian manifold. A subset $S \subseteq M$ is called causally convex if $J_{M}^{+}(S) \cap J_{M}^{-}(S) \subseteq S$. Two subsets $S, S^{\prime} \subseteq M$ are called causally disjoint if $\left(J_{M}^{+}(S) \cup J_{M}^{-}(S)\right) \cap S^{\prime}=\emptyset$.

Remark 2.2. In words, a subset $S \subseteq M$ is causally convex if every causal curve that starts and ends in $S$ is contained entirely in $S$. Two subsets $S, S^{\prime} \subseteq M$ are causally disjoint if there exists no causal curve in $M$ connecting $S$ and $S^{\prime}$.

Definition 2.3. A time-oriented Lorentzian manifold $M$ is called globally hyperbolic if it admits a Cauchy surface, i.e. a subset $\Sigma \subset M$ that is met exactly once by each inextensible time-like curve in $M$.

The following category of Lorentzian manifolds plays a fundamental role in algebraic quantum field theory, see e.g. [BFV03,FV12,BDFY15,BSW17].

Definition 2.4. We denote by Loc the category whose objects are all oriented and timeoriented globally hyperbolic Lorentzian manifolds $M$ and morphisms are all orientation and time-orientation preserving isometric embeddings $f: M \rightarrow N$ with causally convex and open image $f(M) \subseteq N$.

We introduce the following terminology to specify important (tuples of) Locmorphisms that enter the definitions of algebraic quantum field theories and factorization algebras.

Definition 2.5. (a) A Loc-morphism $f: M \rightarrow N$ is called a Cauchy morphism if its image $f(M) \subseteq N$ contains a Cauchy surface of $N$. We shall write $f: M \stackrel{c}{\rightarrow} N$ for Cauchy morphisms.

(b) A pair of Loc-morphisms $\left(f_{1}: M_{1} \rightarrow N, f_{2}: M_{2} \rightarrow N\right)$ to a common target is called causally disjoint if the images $f_{1}\left(M_{1}\right) \subseteq N$ and $f_{2}\left(M_{2}\right) \subseteq N$ are causally disjoint subsets of $N$. We shall write $f_{1} \perp f_{2}$ for causally disjoint morphisms.

(c) A tuple of Loc-morphisms $\left(f_{1}: M_{1} \rightarrow N, \ldots, f_{n}: M_{n} \rightarrow N\right)$ to a common target is called pairwise disjoint if the images $f_{i}\left(M_{i}\right) \subseteq N$ are pairwise disjoint subsets of $N$, i.e. $f_{i}\left(M_{i}\right) \cap f_{j}\left(M_{j}\right)=\emptyset$, for all $i \neq j$. We shall write $\underline{f}: \underline{M} \rightarrow N$ for tuples $\underline{f}=\left(f_{1}, \ldots, f_{n}\right)$ of pairwise disjoint morphisms.

Remark 2.6. By convention, a 1-tuple $f=(f): \underline{M} \rightarrow N$ of pairwise disjoint morphisms is just a Loc-morphism $f: \bar{M} \rightarrow N$ and there exists a unique empty tuple $\emptyset \rightarrow N$ for each $N \in$ Loc. 
2.2. Factorization algebras. Factorization algebras are typically considered in the context of topological, complex or Riemannian manifolds, see [CG17] for a detailed study. In order to obtain a meaningful comparison to algebraic quantum field theory, which is typically considered in the context of globally hyperbolic Lorentzian manifolds, we shall introduce below a variant of factorization algebras on the category Loc from Definition 2.4. A similar concept of factorization algebras on Loc appeared before in [GR17]. For what follows let us fix any cocomplete closed symmetric monoidal category $(\mathbf{C}, \otimes, I, \tau)$, e.g. the category of vector spaces $\mathbf{V e c}_{\mathbb{K}}$ over a field $\mathbb{K}$.

A prefactorization algebra $\mathfrak{F}$ on Loc with values in $\mathbf{C}$ is given by the following data:

(i) for each $M \in \mathbf{L o c}$, an object $\mathfrak{F}(M) \in \mathbf{C}$;

(ii) for each tuple $f=\left(f_{1}, \ldots, f_{n}\right): \underline{M} \rightarrow N$ of pairwise disjoint morphisms, a C-morphism $\mathfrak{F}(f): \bigotimes_{i=1}^{n} \mathfrak{F}\left(M_{i}\right) \rightarrow \mathfrak{F}(N)$ (called factorization product), with the convention that to the empty tuple $\emptyset \rightarrow N$ is assigned a morphism $I \rightarrow \mathfrak{F}(N)$ from the monoidal unit.

These data are required to satisfy the following conditions:

1. for every $\underline{f}=\left(f_{1}, \ldots, f_{n}\right): \underline{M} \rightarrow N$ and $\underline{g}_{i}=\left(g_{i 1}, \ldots, g_{i k_{i}}\right): \underline{L}_{i} \rightarrow M_{i}$, for $i=1, \ldots, n$, the diagram

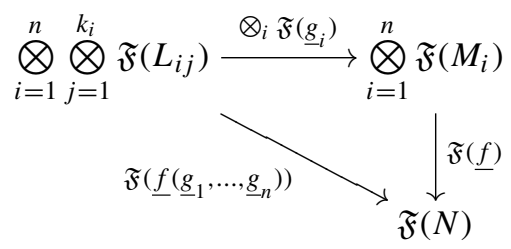

in C commutes, where $\underline{f}\left(\underline{g}_{1}, \ldots, \underline{g}_{n}\right):=\left(f_{1} g_{11}, \ldots, f_{n} g_{n k_{n}}\right):\left(\underline{L}_{1}, \ldots, \underline{L}_{n}\right) \rightarrow N$ is given by composition in Loc;

2. for every $M \in$ Loc, $\mathfrak{F}\left(\operatorname{id}_{M}\right)=\operatorname{id}_{\mathfrak{F}(M)}: \mathfrak{F}(M) \rightarrow \mathfrak{F}(M)$;

2. for every $f=\left(f_{1}, \ldots, f_{n}\right): \underline{M} \rightarrow N$ and every permutation $\sigma \in \Sigma_{n}$, the diagram

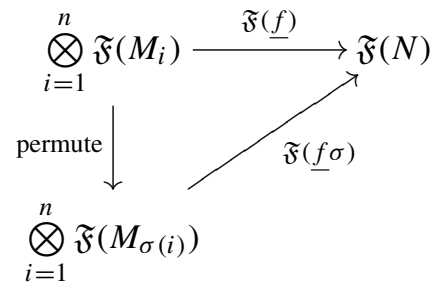

in $\mathbf{C}$ commutes, where $f \sigma:=\left(f_{\sigma(1)}, \ldots, f_{\sigma(n)}\right): \underline{M} \sigma \rightarrow N$ is given by right permutation.

A morphism $\zeta: \mathfrak{F} \rightarrow \mathfrak{G}$ of prefactorization algebras is a family $\zeta_{M}: \mathfrak{F}(M) \rightarrow \mathfrak{G}(M)$ of $\mathbf{C}$-morphisms, for all $M \in \mathbf{L o c}$, that is compatible with the factorization products, 
i.e. for all $\underline{f}: \underline{M} \rightarrow N$ the diagram

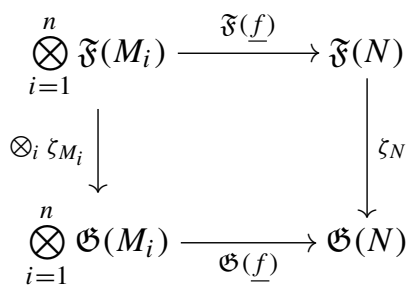

in $\mathbf{C}$ commutes.

Definition 2.7. We denote by PFA the category of prefactorization algebras on Loc.

Factorization algebras are prefactorization algebras that satisfy a suitable descent condition with respect to Weiss covers [CG17]. For proving our results in this paper, it is sufficient to assume a weaker descent condition that we shall call additivity in reference to a similar property in algebraic quantum field theory [Few13]. As explained below, this includes in particular all factorization algebras on Loc. Before we can formalize the additivity property, we have to introduce some further terminology and notations.

Definition 2.8. For $M \in$ Loc, we denote by $\mathbf{R C}_{M}$ the category of all relatively compact and causally convex open subsets $U \subseteq M$ with morphisms given by subset inclusions.

Remark 2.9. Note that the assignment $M \mapsto \mathbf{R C}_{M}$ may be promoted to a functor $\mathbf{R C}_{(-)}$: Loc $\rightarrow$ Cat with values in the category of (small) categories. Concretely, given any Locmorphism $f: M \rightarrow N$, then the functor $\mathbf{R C}_{f}: \mathbf{R C}_{M} \rightarrow \mathbf{R C}_{N}$ sends each relatively compact and causally convex open subset $U \subseteq M$ to its image $f(U) \subseteq N$. Since $f$ is continuous, it follows that this is a relatively compact and causally convex open subset of $N$.

Lemma 2.10. For every $M \in \mathbf{L o c}$, the category $\mathbf{R C}_{M}$ is a directed set.

Proof. Let $U_{1}, U_{2} \in \mathbf{R C}_{M}$. We shall construct $U \in \mathbf{R C}_{M}$ such that $U_{i} \subseteq U$, for $i=1,2$. Since $K:=\overline{U_{1}} \cup \overline{U_{2}}$ is compact, there exists a Cauchy surface $\Sigma$ of $M$ such that $K \subseteq I_{M}^{-}(\Sigma)$. We set $S:=J_{M}^{+}(K) \cap J_{M}^{-}(\Sigma)$ and observe that this is a compact subset of $M$ by [BGP07, Corollary A.5.4]. Using also [BGP07, Lemma A.5.12], it follows that $U:=I_{M}^{+}(K) \cap I_{M}^{-}(S)$ belongs to $\mathbf{R C}_{M}$. By construction, $U$ contains both $U_{1}$ and $U_{2}$.

We may restrict the orientation, time-orientation and metric on $M$ to the causally convex open subsets $U \in \mathbf{R C}_{M}$ and thereby define objects $U \in$ Loc. Every inclusion $U \subseteq V$ in $\mathbf{R C}_{M}$ then defines a Loc-morphism $\iota_{U}^{V}: U \rightarrow V$. Hence, we can regard $\mathbf{R C}_{M} \subseteq$ Loc as a subcategory, for every $M \in$ Loc, and restrict any prefactorization algebra $\mathfrak{F} \in$ PFA to a functor $\left.\mathfrak{F}\right|_{M}: \mathbf{R C}_{M} \rightarrow \mathbf{C}$.

Definition 2.11. A prefactorization algebra $\mathfrak{F} \in$ PFA is called additive if, for every $M \in \mathbf{L o c}$, the canonical morphism

$$
\operatorname{colim}\left(\left.\mathfrak{F}\right|_{M}: \mathbf{R C}_{M} \rightarrow \mathbf{C}\right) \stackrel{\cong}{\longrightarrow} \mathfrak{F}(M)
$$

is an isomorphism in $\mathbf{C}$. We denote by $\mathbf{P F A}{ }^{\text {add }} \subseteq$ PFA the full subcategory of additive prefactorization algebras. 
Remark 2.12. The additivity condition formalizes the idea that $\mathfrak{F}(M)$ is "generated" by the images of the maps $\mathfrak{F}(U) \rightarrow \mathfrak{F}(M)$, for all relatively compact and causally convex open subsets $U \subseteq M$. Interpreting $\mathfrak{F}(M)$ as a collection of observables for a quantum field theory, this means that all observables described by $\mathfrak{F}(M)$ arise from relatively compact regions $U \subseteq M$.

Proposition 2.13. Every factorization algebra $\mathfrak{F}$ on $\mathbf{L o c}$ is an additive prefactorization algebra.

Proof. Suppose that $\mathfrak{F}$ is a factorization algebra [CG17], i.e. it satisfies a cosheaf condition with respect to all Weiss covers of every $M \in$ Loc. For every $M \in$ Loc, the cover defined by $\mathbf{R} \mathbf{C}_{M}$ is a Weiss cover. Indeed, given finitely many points $x_{1}, \ldots, x_{n} \in M$, there exist $U_{i} \in \mathbf{R C}_{M}$ with $x_{i} \in U_{i}$ and hence $U \in \mathbf{R C}_{M}$ with $x_{1}, \ldots, x_{n} \in U$ because $\mathbf{R C}_{M}$ is directed by Lemma 2.10 . The property of being a factorization algebra then implies that the canonical diagram

$$
\bigsqcup_{\substack{U, V \in \mathbf{R} \mathbf{C}_{M} \\ U \cap V \neq \emptyset}} \mathfrak{F}(U \cap V) \Longrightarrow \underset{U \in \mathbf{R C}_{M}}{\coprod_{M}} \mathfrak{F}(U) \longrightarrow \mathfrak{F}(M)
$$

is a coequalizer in C. Our claim then follows by observing that the cocones of (2.4) are canonically identified with the cocones of (2.5). Indeed, any cocone $\left\{\alpha_{U}: \mathfrak{F}(U) \rightarrow Z\right\}$ of (2.4) defines a cocone of (2.5) because $U \cap V \in \mathbf{R C}_{M}$ (whenever nonempty) and hence the diagram

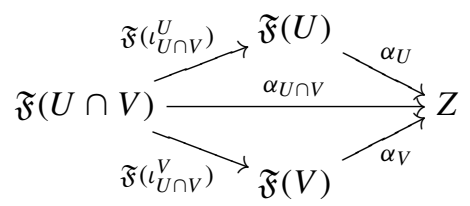

in $\mathbf{C}$ commutes. Vice versa, any cocone $\left\{\alpha_{U}: \mathfrak{F}(U) \rightarrow Z\right\}$ of (2.5) defines a cocone of (2.4) because $U \cap V=U$, for all $U \subseteq V$, and hence the diagram

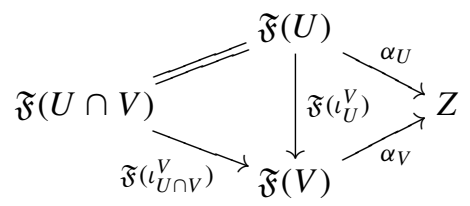

in $\mathbf{C}$ commutes.

As a last definition, we would like to introduce a suitable local constancy property that is adapted to the category Loc. This property will play a crucial role in establishing our comparison results. Recall from Definition 2.5 the concept of Cauchy morphisms.

Definition 2.14. A prefactorization algebra $\mathfrak{F} \in$ PFA is called Cauchy constant if $\mathfrak{F}(f)$ : $\mathfrak{F}(M) \rightarrow \mathfrak{F}(N)$ is an isomorphism in $\mathbf{C}$, for every Cauchy morphism $f: M \stackrel{c}{\rightarrow} N$. We denote by $\mathbf{P F A}^{c} \subseteq$ PFA the full subcategory of Cauchy constant prefactorization algebras. The full subcategory $\mathbf{P F A}{ }^{\text {add }, c} \subseteq \mathbf{P F A}^{\text {add }}$ of Cauchy constant additive prefactorization algebras is defined analogously. 
2.3. Algebraic quantum field theories. Let $\mathbf{C}$ be a cocomplete closed symmetric monoidal category as in the previous subsection. We briefly review the basic definitions for $\mathbf{C}$ valued algebraic quantum field theories on Loc following [BSW17]. Algebraic quantum field theories with $*$-involutions are defined later in Sect. 5.2. We also refer to [BFV03, FV12, BDFY15] for a broader introduction to algebraic quantum field theories and their applications to physics.

Let us denote by $\mathbf{A l g}:=\mathbf{A l g}_{\text {As }}(\mathbf{C})$ the category of associative and unital algebras in $\mathbf{C}$. An algebraic quantum field theory $\mathfrak{A}$ on Loc with values in $\mathbf{C}$ is a functor $\mathfrak{A}: \mathbf{L o c} \rightarrow \mathbf{A l g}$ that satisfies the Einstein causality axiom: for every pair of causally disjoint morphisms $\left(f_{1}: M_{1} \rightarrow N\right) \perp\left(f_{2}: M_{2} \rightarrow N\right)$, the diagram

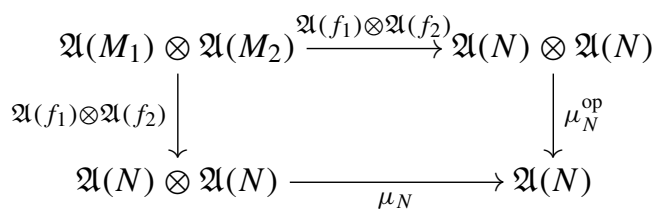

in $\mathbf{C}$ commutes, where $\mu_{N}^{(\mathrm{op})}$ denotes the (opposite) multiplication on $\mathfrak{A}(N)$. A morphism $\kappa: \mathfrak{A} \rightarrow \mathfrak{B}$ of algebraic quantum field theories is a natural transformation between the underlying functors.

Definition 2.15. We denote by AQFT the category of algebraic quantum field theories on Loc.

For proving some of the results of this paper, we require a relatively mild variant of an additivity property in the sense of [Few13]. Recall from Definition 2.8 the category $\mathbf{R C}_{M}$ of relatively compact and causally convex open subsets of $M \in$ Loc.

Definition 2.16. An algebraic quantum field theory $\mathfrak{A} \in \mathbf{A Q F T}$ is called additive if, for every $M \in \mathbf{L o c}$, the canonical morphism

$$
\operatorname{colim}\left(\left.\mathfrak{A}\right|_{M}: \mathbf{R C}_{M} \rightarrow \mathbf{A l g}\right) \stackrel{\cong}{\longrightarrow} \mathfrak{A}(M)
$$

is an isomorphism in Alg. We denote by AQFT ${ }^{\text {add }} \subseteq$ AQFT the full subcategory of additive algebraic quantum field theories.

Remark 2.17. Because $\mathbf{R C}_{M}$ is a directed set by Lemma 2.10, the colimit in Definition 2.16 can be computed in the underlying category $\mathbf{C}$, see e.g. [Fre17, Proposition 1.3.6]. Hence, to check if an algebraic quantum field theory $\mathfrak{A} \in \mathbf{A Q F T}$ is additive, one can consider its underlying functor $\mathfrak{A}:$ Loc $\rightarrow \mathbf{C}$ to the category $\mathbf{C}$ (i.e. forget the algebra structures) and equivalently check if colim $\left(\left.\mathfrak{A}\right|_{M}: \mathbf{R C}_{M} \rightarrow \mathbf{C}\right) \rightarrow \mathfrak{A}(M)$ is an isomorphism in $\mathbf{C}$. $\triangle$

Furthermore, we introduce a suitable local constancy property that is also known in the literature as the time-slice axiom.

Definition 2.18. An algebraic quantum field theory $\mathfrak{A} \in \mathbf{A Q F T}$ is called Cauchy constant if $\mathfrak{A}(f): \mathfrak{A}(M) \rightarrow \mathfrak{A}(N)$ is an isomorphism in $\mathbf{A l g}$, for every Cauchy morphism $f: M \stackrel{c}{\rightarrow} N$. We denote by AQFT ${ }^{c} \subseteq$ AQFT the full subcategory of Cauchy constant algebraic quantum field theories. The full subcategory AQFT ${ }^{\text {add }}, c \subseteq \mathbf{A Q F T}^{\text {add }}$ of Cauchy constant additive algebraic quantum field theories is defined analogously. 


\section{From PFA to AQFT}

In this section we show that every Cauchy constant additive prefactorization algebra $\mathfrak{F} \in$ PFA $^{\text {add }, c}$ (cf. Definitions 2.11 and 2.14) defines a Cauchy constant additive algebraic quantum field theory (cf. Definitions 2.16 and 2.18). This construction will define a functor $\mathbb{A}: \mathbf{P F A}^{\text {add }, c} \rightarrow \mathbf{A Q F T}^{\text {add }, c}$.

Our construction consists of three steps, which will be carried out in detail in individual subsections below. Step (1) consists of proving that, for each $M \in$ Loc, the object $\mathfrak{F}(M) \in \mathbf{C}$ carries canonically the structure of an associative and unital algebra in $\mathbf{C}$. This step relies on Cauchy constancy, while it does not require that the additivity property holds true. Step (2) consists of proving that these algebra structures are compatible with the maps $\mathfrak{F}(f): \mathfrak{F}(M) \rightarrow \mathfrak{F}(N)$ induced by Loc-morphisms $f: M \rightarrow N$. Here our additivity property turns out to be crucial. Finally, in step (3) we show that the resulting functor Loc $\rightarrow$ Alg satisfies the properties of a Cauchy constant additive algebraic quantum field theory, cf. Sect. 2.3.

3.1. Object-wise algebra structure. All results of this subsection do not use the additivity property from Definition 2.11 . Hence, we let $\mathfrak{F} \in \mathbf{P F A}^{c}$ be any Cauchy constant prefactorization algebra.

Let us fix any $M \in$ Loc. The basic idea to define a multiplication map $\mu_{M}: \mathfrak{F}(M) \otimes$ $\mathfrak{F}(M) \rightarrow \mathfrak{F}(M)$ is as follows: Consider two causally convex open subsets $U_{+}, U_{-} \subseteq M$ satisfying (i) there exists a Cauchy surface $\Sigma$ of $M$ such that $U_{ \pm} \subseteq I_{M}^{ \pm}(\Sigma)$, and (ii) $\iota_{U_{ \pm}}^{M}$ : $U_{ \pm} \stackrel{c}{\rightarrow} M$ are Cauchy morphisms. In particular, $U_{+} \cap U_{-}=\emptyset$ are disjoint and hence provide a pair of disjoint morphisms $\iota_{\underline{U}}^{M}=\left(\iota_{U_{+}}^{M}, \iota_{U_{-}}^{M}\right): \underline{U} \rightarrow M$. We define $\mu_{M}$ by the commutative diagram

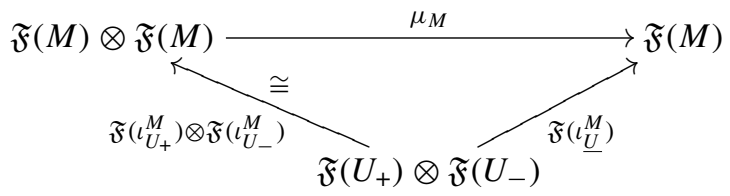

where the upward-left pointing arrow is an isomorphism because $\mathfrak{F}$ is by hypothesis Cauchy constant. A priori, it is not clear whether different choices of such $\iota_{U}^{M}: \underline{U} \rightarrow M$ lead to the same multiplication map in (3.1). The possible choices are recorded in the following category.

Definition 3.1. Let $M \in$ Loc. We denote by $\mathbf{P}_{M}$ the category whose objects are all pairs of disjoint morphisms $\iota_{\underline{U}}^{M}=\left(\iota_{U_{+}}^{M}, \iota_{U_{-}}^{M}\right): \underline{U} \rightarrow M$ corresponding to causally convex open subsets $U_{+}, U_{-} \subseteq \bar{M}$ that satisfy

(i) there exists a Cauchy surface $\Sigma$ of $M$ such that $U_{ \pm} \subseteq I_{M}^{ \pm}(\Sigma)$, and

(ii) $\iota_{U_{ \pm}}^{M}: U_{ \pm} \stackrel{c}{\rightarrow} M$ are Cauchy morphisms.

There exists a unique morphism $\left(\iota_{\underline{U}}^{M}: \underline{U} \rightarrow M\right) \rightarrow\left(\iota_{\underline{V}}^{M}: \underline{V} \rightarrow M\right)$ if and only if $U_{ \pm} \subseteq V_{ \pm}$

Lemma 3.2. For every $M \in \mathbf{L o c}$, the category $\mathbf{P}_{M}$ is non-empty and connected. 
Proof. Non-empty Choose any Cauchy surface $\Sigma$ of $M$ and define $\Sigma_{ \pm}:=I_{M}^{ \pm}(\Sigma)$. Then $\iota_{\underline{\Sigma}}^{M}=\left(\iota_{\Sigma_{+}}^{M}, \iota_{\Sigma_{-}}^{M}\right): \underline{\Sigma} \rightarrow M$ defines an object in $\mathbf{P}_{M}$.

Connected We have to prove that there exists a zig-zag of morphisms in $\mathbf{P}_{M}$ between every pair of objects $\iota_{\underline{U}}^{M}: \underline{U} \rightarrow M$ and $\iota_{\underline{V}}^{M}: \underline{V} \rightarrow M$. For every object $\iota_{\underline{U}}^{M}: \underline{U} \rightarrow M$ in $\mathbf{P}_{M}$, there exists by hypothesis a Cauchy surface $\Sigma$ of $M$ such that $U_{ \pm} \subseteq \bar{\Sigma}_{ \pm}:=I_{M}^{ \pm}(\Sigma)$. Hence, there exists a morphism $\left(\iota_{\underline{U}}^{M}: \underline{U} \rightarrow M\right) \rightarrow\left(\iota_{\underline{\Sigma}}^{M}: \underline{\Sigma} \rightarrow M\right)$. As a consequence, our original problem reduces to finding a zig-zag of morphisms in $\mathbf{P}_{M}$ between $\iota_{\underline{\Sigma}}^{M}$ : $\underline{\Sigma} \rightarrow M$ and $\iota_{\underline{\Sigma}^{\prime}}^{M}: \underline{\Sigma}^{\prime} \rightarrow M$, for any two Cauchy surfaces $\Sigma, \Sigma^{\prime}$ of $M$. To exhibit such a zig-zag, let us introduce $\widetilde{U}_{+}:=\Sigma_{+} \cap \Sigma_{+}^{\prime}$ and $\widetilde{U}_{-}:=\Sigma_{-} \cap \Sigma_{-}^{\prime}$. If we could prove that $\iota \stackrel{M}{\widetilde{U}_{ \pm}}: \widetilde{U}_{ \pm} \stackrel{c}{\rightarrow} M$ are Cauchy morphisms, then

$$
\left(\iota_{\underline{\Sigma}}^{M}: \underline{\Sigma} \rightarrow M\right) \longleftarrow\left(\iota_{\underline{\widetilde{U}}}^{M}: \underline{\tilde{U}} \rightarrow M\right) \longrightarrow\left(\iota_{\underline{\Sigma^{\prime}}}^{M}: \underline{\Sigma^{\prime}} \rightarrow M\right)
$$

would provide a zig-zag that proves connectedness of $\mathbf{P}_{M}$.

It remains to show that $\widetilde{U}_{+}=\Sigma_{+} \cap \Sigma_{+}^{\prime}=I_{M}^{+}(\Sigma) \cap I_{M}^{+}\left(\Sigma^{\prime}\right) \subseteq M$ contains a Cauchy surface of $M$. (A similar argument shows that $\widetilde{U}_{-} \subseteq M$ also contains a Cauchy surface of $M$.) Because $\Sigma, \Sigma^{\prime}$ are by hypothesis Cauchy surfaces of $M$, there exists a Cauchy surface $\Sigma_{1} \subset I_{M}^{+}(\Sigma)$ of $M$ in the future of $\Sigma$ and a Cauchy surface $\Sigma_{1}^{\prime} \subset I_{M}^{+}\left(\Sigma^{\prime}\right)$ of $M$ in the future of $\Sigma^{\prime}$. We define the subset

$$
\widetilde{\Sigma}:=\left(\Sigma_{1} \cap J_{M}^{+}\left(\Sigma_{1}^{\prime}\right)\right) \cup\left(J_{M}^{+}\left(\Sigma_{1}\right) \cap \Sigma_{1}^{\prime}\right) \subset \widetilde{U}_{+} \subseteq M
$$

and claim that $\widetilde{\Sigma}$ is a Cauchy surface of $M$. To prove the last statement, consider any inextensible time-like curve $\gamma: I \rightarrow M$, which we may assume without loss of generality to be future directed. (If $\gamma$ would be past directed, then change the orientation of the interval I.) Because $\Sigma_{1}$ and $\Sigma_{1}^{\prime}$ are Cauchy surfaces of $M$, there exist unique $t, t^{\prime} \in I$ such that $\gamma(t) \in \Sigma_{1}$ and $\gamma\left(t^{\prime}\right) \in \Sigma_{1}^{\prime}$. If $t \geq t^{\prime}$, then $\gamma(t) \in \Sigma_{1} \cap J_{M}^{+}\left(\Sigma_{1}^{\prime}\right) \subseteq \widetilde{\Sigma}$, and if $t^{\prime} \geq t$, then $\gamma\left(t^{\prime}\right) \in J_{M}^{+}\left(\Sigma_{1}\right) \cap \Sigma_{1}^{\prime} \subseteq \widetilde{\Sigma}$. Hence, $\gamma$ meets $\widetilde{\Sigma} \subset M$ at least once. Multiple intersections are excluded by the definition of $\widetilde{\Sigma}$ in (3.3) and the fact that both $\Sigma_{1}$ and $\Sigma_{1}^{\prime}$ are Cauchy surfaces of $M$.

Corollary 3.3. For every $M \in$ Loc, the multiplication map $\mu_{M}$ in (3.1) does not depend on the choice of object $\iota_{\underline{U}}^{M}: \underline{U} \rightarrow M$ in $\mathbf{P}_{M}$.

Proof. By Lemma 3.2, it is sufficient to prove that $\iota_{U}^{M}: \underline{U} \rightarrow M$ and $\iota_{V}^{M}: \underline{V} \rightarrow M$ define the same multiplication if $U_{+} \subseteq V_{+}$and $U_{-} \subseteq \overline{V_{-}}$. This is a consequence of the commutative diagram

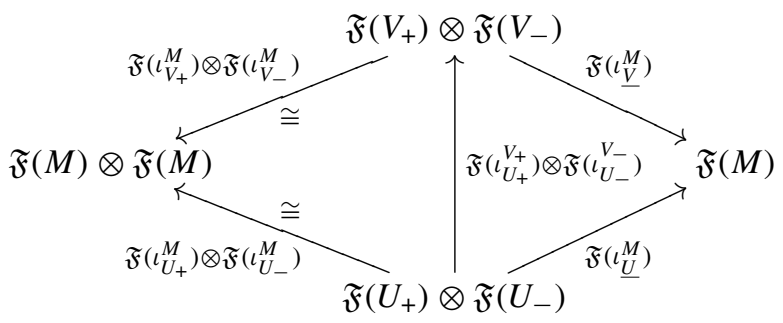

where one also uses the composition properties (2.1) of prefactorization algebras. 
To obtain a unit for $\mathfrak{F}(M)$, we recall that there exists a unique empty tuple of disjoint morphisms $\emptyset \rightarrow M$ to which the prefactorization algebra assigns a $\mathbf{C}$-morphism that we shall denote by $\eta_{M}: I \rightarrow \mathfrak{F}(M)$. The main result of this subsection is as follows.

Proposition 3.4. Let $\mathfrak{F} \in \mathbf{P F A}^{c}$ be any Cauchy constant prefactorization algebra. For every $M \in \mathbf{L o c}$, the object $\mathfrak{F}(M) \in \mathbf{C}$ carries the structure of an associative and unital algebra in $\mathbf{C}$ with multiplication $\mu_{M}: \mathfrak{F}(M) \otimes \mathfrak{F}(M) \rightarrow \mathfrak{F}(M)$ given by (3.1) and unit $\eta_{M}: I \rightarrow \mathfrak{F}(M)$ given by evaluating $\mathfrak{F}$ on the empty tuple $\emptyset \rightarrow M$.

Proof. To prove that the multiplication $\mu_{M}$ is associative, we consider two Cauchy surfaces $\Sigma_{0}, \Sigma_{1}$ of $M$ such that $\Sigma_{1} \subset I_{M}^{+}\left(\Sigma_{0}\right)$, i.e. $\Sigma_{1}$ is in the future of $\Sigma_{0}$. Using the independence result from Corollary 3.3 and the composition properties of prefactorization algebras from Sect. 2.2, one easily confirms that $\mu_{M}$ (id $\otimes \mu_{M}$ ) is the upper path and $\mu_{M}\left(\mu_{M} \otimes\right.$ id) the lower path from $\mathfrak{F}(M)^{\otimes 3}$ to $\mathfrak{F}(M)$ in the commutative diagram

$$
\begin{aligned}
& \mathfrak{F}\left(\Sigma_{1+}\right) \otimes \mathfrak{F}\left(\Sigma_{1-} \cap \Sigma_{0+}\right) \otimes \mathfrak{F}\left(\Sigma_{0-}\right) \stackrel{\operatorname{id} \otimes \mathfrak{F}\left(\iota_{\left.\Sigma_{1-} \cap \Sigma_{0+},{ }^{\nu_{1-}} \Sigma_{\Sigma_{0-}}\right)}^{\longrightarrow}\right.}{\longrightarrow} \mathfrak{F}\left(\Sigma_{1+}\right) \otimes \mathfrak{F}\left(\Sigma_{1-}\right) \\
& \mathfrak{F}\left(\iota_{\Sigma_{1+}}^{M}\right) \otimes \mathfrak{F}\left(\iota_{\Sigma_{1-} \cap \Sigma_{0+}}^{M}\right) \otimes \mathfrak{F}\left(\iota_{\Sigma_{0-}}^{M}\right) \downarrow \cong \\
& \begin{array}{rr}
\mathfrak{F}(M) \otimes \mathfrak{F}(M) \otimes \mathfrak{F}(M) & \mathfrak{F}(M) \\
\mathfrak{F}\left(\iota_{\Sigma_{1+}}^{M}\right) \otimes \mathfrak{F}\left(l_{\Sigma_{1-}}^{M} \Sigma_{0+}\right. \\
) \otimes \mathfrak{F}\left(l_{\Sigma_{0-}}^{M}\right) \uparrow \cong & \uparrow \mathfrak{F}\left(\iota_{\Sigma_{0+}}^{M}, l_{\Sigma_{0-}}^{M}\right)
\end{array} \\
& \mathfrak{F}\left(\Sigma_{1+}\right) \otimes \mathfrak{F}\left(\Sigma_{1-} \cap \Sigma_{0+}\right) \otimes \mathfrak{F}\left(\Sigma_{0-}\right) \underset{\mathfrak{F}\left(l_{\Sigma_{1+}, l}^{\Sigma_{0+}, \Sigma_{\Sigma_{-}} \cap \Sigma_{0+}}\right) \otimes \text { id }}{\longrightarrow} \mathfrak{F}\left(\Sigma_{0+}\right) \otimes \mathfrak{F}\left(\Sigma_{0-}\right)
\end{aligned}
$$

where as before we denote by $\Sigma_{ \pm}:=I_{M}^{ \pm}(\Sigma) \subseteq M$ the chronological future/past of a Cauchy surface $\Sigma$ of $M$. Unitality of the product follows immediately from the fact that there exists a unique empty tuple $\emptyset \rightarrow N$ for each $N \in$ Loc and the composition properties (2.1) of prefactorization algebras.

3.2. Naturality of algebra structures. The aim of this subsection is to investigate compatibility between the algebra structures from Proposition 3.4 and the maps $\mathfrak{F}(f)$ : $\mathfrak{F}(M) \rightarrow \mathfrak{F}(N)$ induced by Loc-morphisms. For our main statement to be true it will be crucial to assume that $\mathfrak{F} \in \mathbf{P F A}^{\text {add }, c}$ is a Cauchy constant additive prefactorization algebra in the sense of Definitions 2.11 and 2.14. As a first partial result, we prove the following general statement.

Lemma 3.5. Let $\mathfrak{F} \in \mathbf{P F A}^{c}$ be any Cauchy constant prefactorization algebra (not necessarily additive). Let further $f: M \rightarrow N$ be a Loc-morphism such that the image $f(M) \subseteq N$ is relatively compact. Then $\mathfrak{F}(f): \mathfrak{F}(M) \rightarrow \mathfrak{F}(N)$ preserves the multiplications and units from Proposition 3.4, i.e. $\mu_{N}(\mathfrak{F}(f) \otimes \mathfrak{F}(f))=\mathfrak{F}(f) \mu_{M}$ and $\eta_{N}=\mathfrak{F}(f) \eta_{M}$.

Proof. The units are clearly preserved for every Loc-morphism $f: M \rightarrow N$ because composing the unique empty tuple $\emptyset \rightarrow M$ with $f: M \rightarrow N$ yields the unique empty tuple $\emptyset \rightarrow N$.

Let us focus now on the multiplications. Because $f(M) \subseteq N$ is by hypothesis relatively compact, its closure $\overline{f(M)} \subseteq N$ is compact. Let us take any Cauchy surface 
$\Sigma$ of $M$ and note that $\overline{f(\Sigma)} \subseteq N$ is a compact subset. Using further that $f(M) \subseteq N$ is causally convex and that the causality relation induced by time-like curves is open (cf. [ONe83, Lemma 14.3]), it follows that $\overline{f(\Sigma)} \subseteq N$ is achronal, i.e. every time-like curve in $N$ meets this subset at most once. By [BS06, Theorem 3.8], there exists a Cauchy surface $\widetilde{\Sigma}$ of $N$ such that $f(\Sigma) \subseteq \widetilde{\Sigma}$.

Using the Cauchy surfaces constructed above, we can define the multiplication $\mu_{M}$ in terms of $\Sigma_{ \pm}:=I_{M}^{ \pm}(\Sigma)$ and the multiplication $\mu_{N}$ in terms of $\widetilde{\Sigma}_{ \pm}:=I_{N}^{ \pm}(\widetilde{\Sigma})$, cf. (3.1). By construction, $f: M \rightarrow N$ restricts to Loc-morphisms $f_{\Sigma_{ \pm}}^{\widetilde{\Sigma}_{ \pm}}: \Sigma_{ \pm} \rightarrow \widetilde{\Sigma}_{ \pm}$. Our claim that $\mathfrak{F}(f): \mathfrak{F}(M) \rightarrow \mathfrak{F}(N)$ preserves the multiplications then follows by observing that the diagram

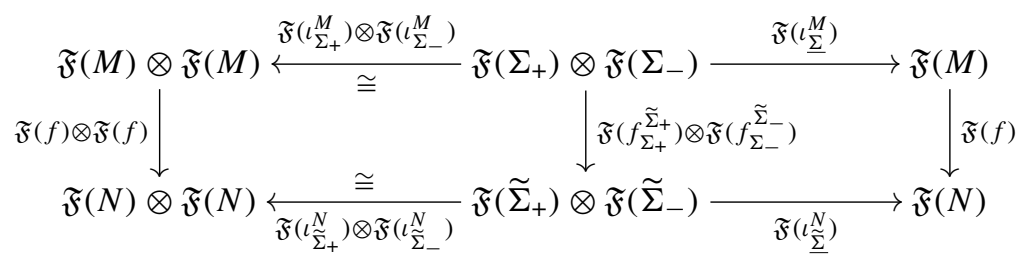

commutes.

Remark 3.6. We would like to emphasize that our assumption that the image $f(M) \subseteq N$ is relatively compact was crucial for the proof of Lemma 3.5. In fact, if one does not assume that the image of the Loc-morphism $f: M \rightarrow N$ is relatively compact, then it is not true that the image $f(\Sigma) \subset N$ of a Cauchy surface $\Sigma$ of $M$ can be extended to a Cauchy surface $\widetilde{\Sigma}$ of $N$. A simple example that demonstrates this feature is given by the subset inclusion $\iota_{U}^{V}: U \rightarrow V$ of the following two diamond regions in 2-dimensional Minkowski spacetime (note that $U$ is not relatively compact as a subset of $V$ ):

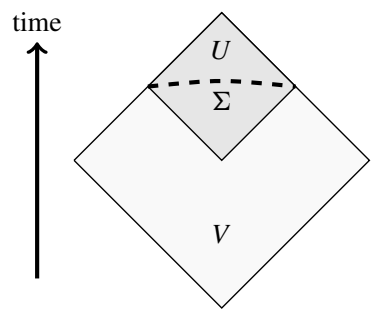

It is evident that no Cauchy surface $\Sigma$ of $U$ admits an extension to a Cauchy surface of $V$. Hence, $\mathfrak{F}\left(\iota_{U}^{V}\right): \mathfrak{F}(U) \rightarrow \mathfrak{F}(V)$ may fail to preserve the multiplications. We shall show below that the issues explained in this remark are solved by considering additive prefactorization algebras as in Definition 2.11.

The main result of this subsection is as follows.

Proposition 3.7. Let $\mathfrak{F} \in \mathbf{P F A}^{\text {add }, c}$ be any Cauchy constant additive prefactorization algebra. For every Loc-morphism $f: M \rightarrow N$, the C-morphism $\mathfrak{F}(f): \mathfrak{F}(M) \rightarrow \mathfrak{F}(N)$ preserves the multiplications and units from Proposition 3.4.

Proof. We already observed in the proof of Lemma 3.5 that $\mathfrak{F}(f)$ preserves the units.

For the multiplications we have to prove that $\mu_{N}(\mathfrak{F}(f) \otimes \mathfrak{F}(f))=\mathfrak{F}(f) \mu_{M}$ as C-morphisms from $\mathfrak{F}(M) \otimes \mathfrak{F}(M)$ to $\mathfrak{F}(N)$. Because $\mathfrak{F}$ is by hypothesis additive (cf. 
Definition 2.11) and the monoidal product $\otimes$ in a cocomplete closed symmetric monoidal category preserves colimits in both entries, it follows that

$$
\mathfrak{F}(M) \otimes \mathfrak{F}(M) \cong \operatorname{colim}_{U, V \in \mathbf{R C}_{M}}(\mathfrak{F}(U) \otimes \mathfrak{F}(V)) \cong \operatorname{colim}_{U \in \mathbf{R C}_{M}}(\mathfrak{F}(U) \otimes \mathfrak{F}(U)),
$$

where in the last step we also used that $\mathbf{R C}_{M}$ is directed by Lemma 2.10. For every $U \in \mathbf{R C}_{M}$, consider the diagram

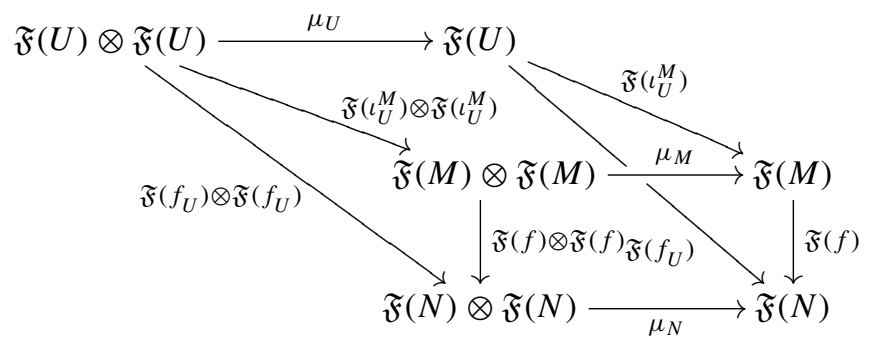

where $f_{U}: U \rightarrow N$ denotes the restriction of $f: M \rightarrow N$ to $U \subseteq M$. The top and bottom squares of this diagram commute because of Lemma 3.5 and the fact that both $U \subseteq M$ and $f(U) \subseteq N$ are relatively compact subsets. The two triangles commute by direct inspection. By universality of the colimit in (3.8), this implies that the front square in (3.9) commutes, proving our claim.

Corollary 3.8. Every Cauchy constant additive prefactorization algebra $\mathfrak{F} \in \mathbf{P F A}^{\text {add }, c}$ defines a functor $\mathbb{A}[\mathfrak{F}]:$ Loc $\rightarrow$ Alg to the category of associative and unital algebras. Explicitly, this functor acts on objects $M \in \operatorname{Loc}$ as $\mathbb{A}[\mathfrak{F}](M):=\left(\mathfrak{F}(M), \mu_{M}, \eta_{M}\right)$ and on Loc-morphisms $f: M \rightarrow N$ as $\mathbb{A}[\mathfrak{F}](f):=\mathfrak{F}(f)$. The assignment $\mathfrak{F} \mapsto \mathbb{A}[\mathfrak{F}]$ canonically extends to a functor $\mathbb{A}: \mathbf{P F A}^{\text {add, } c} \rightarrow \mathbf{A l g}^{\text {Loc }}$, where $\mathbf{A l g}^{\text {Loc }}$ denotes the category of functors from Loc to Alg.

Proof. It remains to prove that every morphism $\zeta: \mathfrak{F} \rightarrow \mathfrak{G}$ in $\mathbf{P F A}{ }^{\text {add }, c}$ defines a natural transformation $\mathbb{A}[\zeta]: \mathbb{A}[\mathfrak{F}] \rightarrow \mathbb{A}[\mathfrak{G}]$ between Alg-valued functors on Loc, i.e. that all components $\zeta_{M}: \mathfrak{F}(M) \rightarrow \mathfrak{G}(M)$ preserve the multiplications and units. For the units this is immediate, while for the multiplications it follows from the fact that the diagram

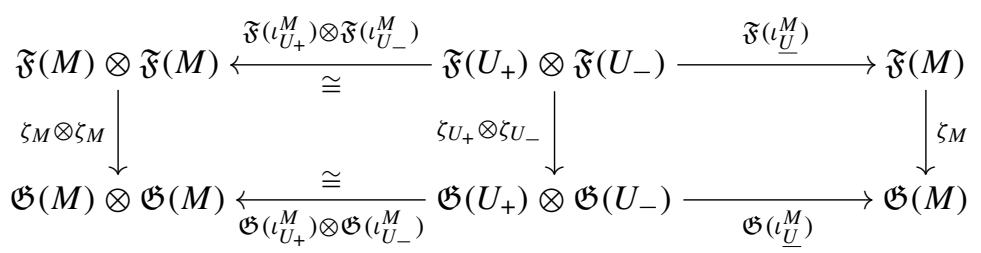

commutes by the compatibility properties (2.3) of prefactorization algebra morphisms.

3.3. Algebraic quantum field theory axioms. The goal of this subsection is to show that the construction above assigns to each Cauchy constant additive prefactorization algebra a Cauchy constant additive algebraic quantum field theory. More precisely, we shall prove that the functor $\mathbb{A}: \mathbf{P F A}^{\text {add, } c} \rightarrow \mathbf{A l g}^{\mathbf{L o c}}$ established in Corollary 3.8 factors through the full subcategory AQFT ${ }^{\text {add }, c} \subseteq \mathbf{A l g}^{\text {Loc }}$ of Cauchy constant additive algebraic quantum field theories. 
Lemma 3.9. Let $\mathfrak{F} \in \mathbf{P F A}^{c}$ be any Cauchy constant prefactorization algebra (not necessarily additive). Let further $\left(f_{1}: M_{1} \rightarrow N\right) \perp\left(f_{2}: M_{2} \rightarrow N\right)$ be any causally disjoint pair of Loc-morphisms such that the images $f_{1}\left(M_{1}\right), f_{2}\left(M_{2}\right) \subseteq N$ are relatively compact. Then $\mu_{N}^{\text {op }}\left(\mathfrak{F}\left(f_{1}\right) \otimes \mathfrak{F}\left(f_{2}\right)\right)=\mu_{N}\left(\mathfrak{F}\left(f_{1}\right) \otimes \mathfrak{F}\left(f_{2}\right)\right)$, where $\mu_{N}^{\text {op })}$ denotes the (opposite) multiplication on $\mathfrak{F}(N)$ from Proposition 3.4.

Proof. In order to compare the two morphisms $\mu_{N}\left(\mathfrak{F}\left(f_{1}\right) \otimes \mathfrak{F}\left(f_{2}\right)\right)$ and $\mu_{N}^{\text {op }}\left(\mathfrak{F}\left(f_{1}\right) \otimes\right.$ $\left.\mathfrak{F}\left(f_{2}\right)\right)$ from $\mathfrak{F}\left(M_{1}\right) \otimes \mathfrak{F}\left(M_{2}\right)$ to $\mathfrak{F}(N)$, we introduce convenient ways to compute these composites. Let us choose arbitrary Cauchy surfaces $\Sigma_{1}$ of $M_{1}$ and $\Sigma_{2}$ of $M_{2}$. As in the proof of Lemma 3.5, we deduce that $\overline{f_{1}\left(\Sigma_{1}\right)}, \overline{f_{2}\left(\Sigma_{2}\right)} \subseteq N$ are achronal compact subsets. Causal disjointness of the pair $f_{1} \perp f_{2}$ entails achronality of the union $\overline{f_{1}\left(\Sigma_{1}\right)} \cup$ $\overline{f_{2}\left(\Sigma_{2}\right)} \subseteq N$. By [BS06, Theorem 3.8], there exists a Cauchy surface $\widetilde{\Sigma}$ of $N$ that contains the union $\overline{f_{1}\left(\Sigma_{1}\right)} \cup \overline{f_{2}\left(\Sigma_{2}\right)} \subseteq \widetilde{\Sigma}$. Similarly, choosing any Cauchy surface $\Sigma_{1}^{\prime} \subset I_{M_{1}}^{+}\left(\Sigma_{1}\right)$ of $M_{1}$ that lies in the future of $\Sigma_{1}$ and any Cauchy surface $\Sigma_{2}^{\prime} \subset I_{M_{2}}^{-}\left(\Sigma_{2}\right)$ of $M_{2}$ that lies in the past of $\Sigma_{2}$, there exists a Cauchy surface $\widetilde{\Sigma}^{\prime}$ of $N$ that contains the union $\overline{f_{1}\left(\Sigma_{1}^{\prime}\right)} \cup \overline{f_{2}\left(\Sigma_{2}^{\prime}\right)} \subseteq \widetilde{\Sigma}^{\prime}$. Let us introduce

$$
U_{1}:=I_{M_{1}}^{+}\left(\Sigma_{1}\right) \cap I_{M_{1}}^{-}\left(\Sigma_{1}^{\prime}\right) \subseteq M_{1}, \quad U_{2}:=I_{M_{2}}^{+}\left(\Sigma_{2}^{\prime}\right) \cap I_{M_{2}}^{-}\left(\Sigma_{2}\right) \subseteq M_{2},
$$

and also consider $\widetilde{\Sigma}_{ \pm}:=I_{N}^{ \pm}(\widetilde{\Sigma}) \subseteq N$ and $\widetilde{\Sigma}_{ \pm}^{\prime}:=I_{N}^{ \pm}\left(\widetilde{\Sigma}^{\prime}\right) \subseteq N$. By construction, $\iota_{U_{i}}^{M_{i}}: U_{i} \stackrel{c}{\rightarrow} M_{i}$, for $i=1,2$, and $\iota_{\widetilde{\Sigma}_{ \pm}^{(\prime)}}^{N}: \widetilde{\Sigma}_{ \pm}^{(\prime)} \stackrel{c}{\rightarrow} N$ are Cauchy morphisms. The following picture illustrates in dark gray the chosen subsets $U_{1} \subseteq M_{1}$ and $U_{2} \subseteq M_{2}$ :

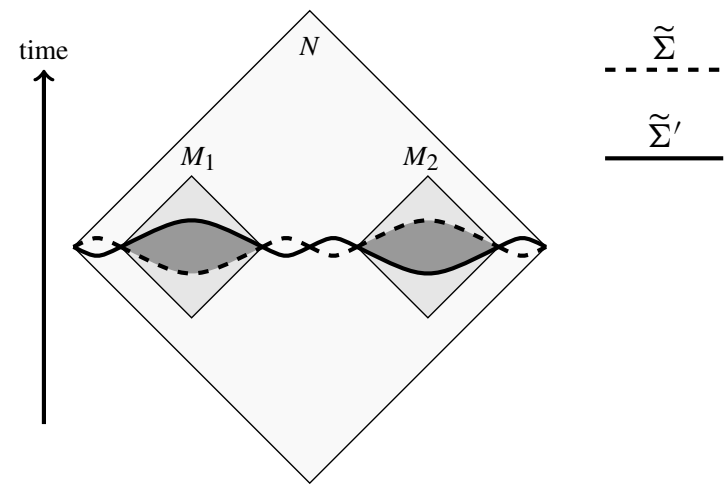

With these preparations, we can compute $\mu_{N}\left(\mathfrak{F}\left(f_{1}\right) \otimes \mathfrak{F}\left(f_{2}\right)\right)$ by

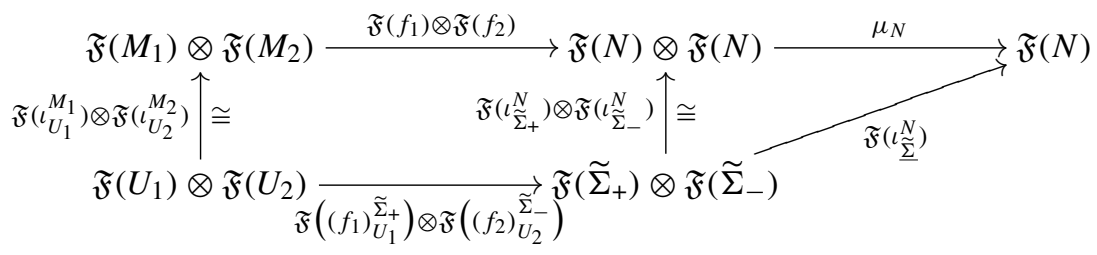


where $\left(f_{1}\right)_{U_{1}}^{\widetilde{\Sigma}_{+}}: U_{1} \rightarrow \widetilde{\Sigma}_{+}$denotes the restriction of $f_{1}: M_{1} \rightarrow N$ to $U_{1} \subseteq M_{1}$, and analogously for $\left(f_{2}\right)_{U_{2}}^{\widetilde{\Sigma}_{-}}$. Similarly, $\mu_{N}^{\text {op }}\left(\mathfrak{F}\left(f_{1}\right) \otimes \mathfrak{F}\left(f_{2}\right)\right)$ can be computed by

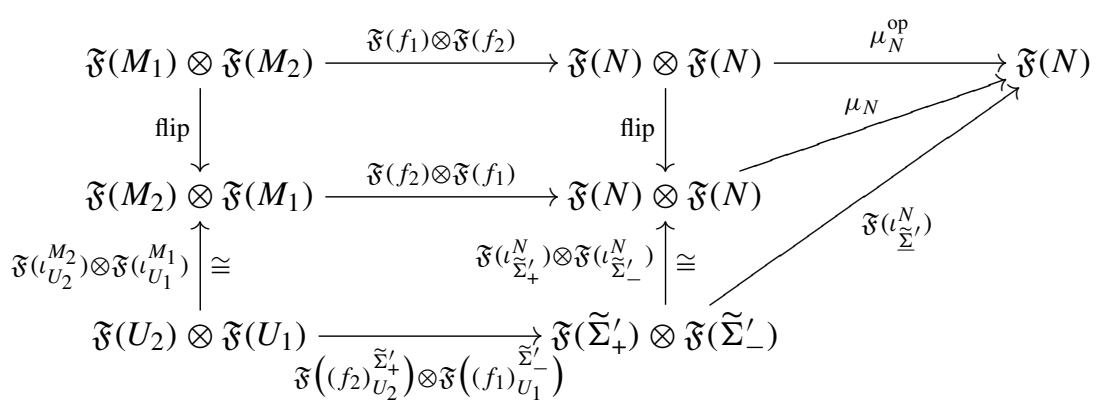

The claim follows from the equivariance property (2.2) of prefactorization algebras.

The main result of this subsection is as follows.

Proposition 3.10. Let $\mathfrak{F} \in \mathbf{P F A}^{\text {add }, c}$ be any Cauchy constant additive prefactorization algebra. Let further $\left(f_{1}: M_{1} \rightarrow N\right) \perp\left(f_{2}: M_{2} \rightarrow N\right)$ be any causally disjoint pair of Loc-morphisms. Then $\mu_{N}^{\text {op }}\left(\mathfrak{F}\left(f_{1}\right) \otimes \mathfrak{F}\left(f_{2}\right)\right)=\mu_{N}\left(\mathfrak{F}\left(f_{1}\right) \otimes \mathfrak{F}\left(f_{2}\right)\right)$, where $\mu_{N}^{\text {(op) }}$ denotes the (opposite) multiplication on $\mathfrak{F}(N)$ from Proposition 3.4.

Proof. Because $\mathfrak{F}$ is by hypothesis additive (cf. Definition 2.11) and the monoidal product $\otimes$ in a cocomplete closed symmetric monoidal category preserves colimits in both entries, it follows that

$$
\mathfrak{F}\left(M_{1}\right) \otimes \mathfrak{F}\left(M_{2}\right) \cong \operatorname{colim}_{\left(U_{1}, U_{2}\right) \in \mathbf{R C}_{M_{1}} \times \mathbf{R C}_{M_{2}}}\left(\mathfrak{F}\left(U_{1}\right) \otimes \mathfrak{F}\left(U_{2}\right)\right) .
$$

For every $\left(U_{1}, U_{2}\right) \in \mathbf{R C}_{M_{1}} \times \mathbf{R C}_{M_{2}}$, consider the diagram

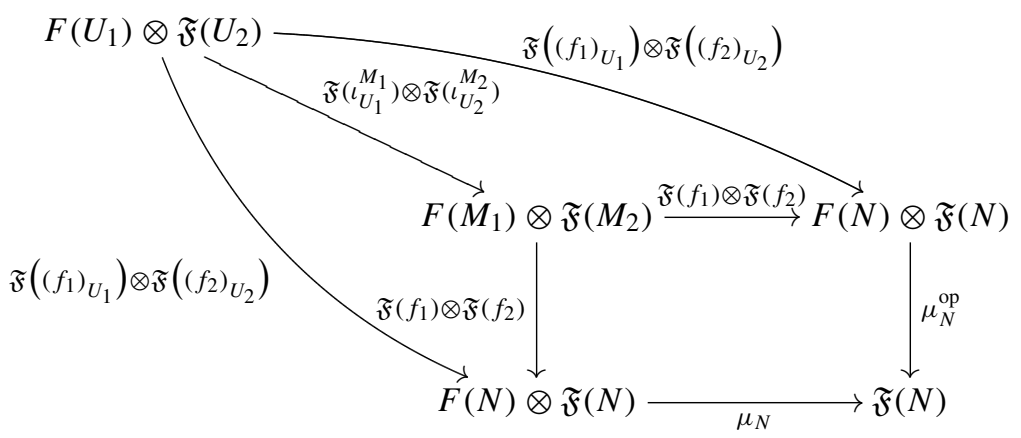

where $\left(f_{i}\right)_{U_{i}}: U_{i} \rightarrow N$ denotes the restriction of $f_{i}: M_{i} \rightarrow N$ to $U_{i} \subseteq M_{i}$, for $i=1,2$. The two triangles coincide and commute by direct inspection. Furthermore, for every $\left(U_{1}, U_{2}\right) \in \mathbf{R C}_{M_{1}} \times \mathbf{R} \mathbf{C}_{M_{2}}$, the outer square commutes as a consequence of Lemma 3.9 applied to the causally disjoint pair $\left(f_{1}\right)_{U_{1}} \perp\left(f_{2}\right)_{U_{2}}$, whose images $f_{1}\left(U_{1}\right), f_{2}\left(U_{2}\right) \subseteq N$ are relatively compact subsets. Hence, by universality of the colimit in (3.15), the inner square commutes as well, which is our claim. 
Proposition 3.10 leads to the following refinement of Corollary 3.8.

Theorem 3.11. Every Cauchy constant additive prefactorization algebra $\mathfrak{F} \in \mathbf{P F A}^{\text {add }, c}$ defines a Cauchy constant additive algebraic quantum field theory $\mathbb{A}[\mathfrak{F}] \in \mathbf{A Q F T}{ }^{\text {add }} c$. Hence, the functor $\mathbb{A}:$ PFA $^{\text {add }, c} \rightarrow \mathbf{A l g}^{\text {Loc }}$ from Corollary 3.8 factors through the full subcategory $\mathbf{A Q F T} \mathbf{T}^{\text {add }, c} \subseteq \mathbf{A l g}^{\text {Loc }}$.

Proof. Proposition 3.10 implies that the functor $\mathbb{A}[\mathfrak{F}]:$ Loc $\rightarrow$ Alg defined in Corollary 3.8 is an algebraic quantum field theory, i.e. it satisfies the Einstein causality axiom (2.7). Because $\mathfrak{F}$ is by hypothesis Cauchy constant, it follows that $\mathbb{A}[\mathfrak{F}]$ is Cauchy constant too. Because the underlying functors $\left.\mathbb{A}[\mathfrak{F}]\right|_{M}=\left.\mathfrak{F}\right|_{M}: \mathbf{R C}_{M} \rightarrow \mathbf{C}$ to the category $\mathbf{C}$ coincide, additivity of $\mathfrak{F} \in \mathbf{P F A}^{\text {add }, c}$ and Remark 2.17 immediately imply additivity of $\mathbb{A}[\mathfrak{F}]$. Hence, $\mathbb{A}[\mathfrak{F}] \in \mathbf{A Q F T}{ }^{\text {add }, c}$.

\section{From AQFT to PFA}

In this section we show that every algebraic quantum field theory $\mathfrak{A} \in \mathbf{A Q F T}$ defines a variant of a prefactorization algebra on Loc where the factorization products are defined only for those tuples of pairwise disjoint morphisms $f: \underline{M} \rightarrow N$ that are in a suitable sense time-orderable. We shall call this type of prefactorization algebras time-orderable and denote the corresponding category by tPFA. Our construction defines a functor $\mathbb{F}:$ AQFT $\rightarrow$ tPFA to the category of time-orderable prefactorization algebras. Cauchy constancy and additivity do not play a role in this section, however we shall prove that these properties are preserved by our functor.

Let $\mathfrak{A} \in \mathbf{A Q F T}$ be an algebraic quantum field theory. Our aim is to construct from this data factorization products $\mathbb{F}[\mathfrak{A}](f): \bigotimes_{i=1}^{n} \mathfrak{A}\left(M_{i}\right) \rightarrow \mathfrak{A}(N)$, for suitable tuples of pairwise disjoint morphisms $f=\left(\overline{f_{1}}, \ldots, f_{n}\right): \underline{M} \rightarrow N$. For $n=0$, i.e. the empty tuples $\emptyset \rightarrow N$, we may take the unit $\eta_{N}: I \rightarrow \mathfrak{A}(N)$ of the associative and unital algebra $\mathfrak{A}(N)$ that is assigned by $\mathfrak{A}$ to $N \in$ Loc. For $n=1$, the tuples of pairwise disjoint morphisms are just Loc-morphisms $f: M \rightarrow N$, hence we may take the $\mathbf{C}$ morphism $\mathbb{F}[\mathfrak{A}](f):=\mathfrak{A}(f): \mathfrak{A}(M) \rightarrow \mathfrak{A}(N)$ that is obtained from the Alg-morphism assigned by $\mathfrak{A}$ to $f: M \rightarrow N$ via the forgetful functor Alg $\rightarrow \mathbf{C}$. For $n \geq 2$, the envisaged construction becomes far less obvious. Let us consider for the moment $n=2$ and a pair of disjoint morphisms $\underline{f}=\left(f_{1}, f_{2}\right): \underline{M} \rightarrow N$. Inspired by our previous construction (3.1) of multiplications from factorization products, we propose to define $\mathbb{F}[\mathfrak{A}](f): \mathfrak{A}\left(M_{1}\right) \otimes \mathfrak{A}\left(M_{2}\right) \rightarrow \mathfrak{A}(N)$ by the commutative diagram

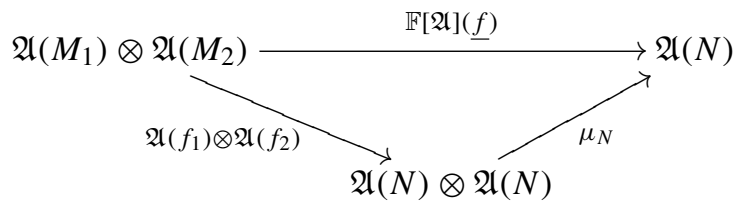

in $\mathbf{C}$. This is however problematic in view of the equivariance property (2.2) of prefactorization algebras. In fact, if we used (4.1) for all pairs of disjoint morphisms $\underline{f}=\left(f_{1}, f_{2}\right): \underline{M} \rightarrow N$, then (2.2) would be satisfied if and only if the diagram in (2.7) commutes, which is in general not the case unless $f_{1} \perp f_{2}$ are causally disjoint. By closer inspection of (3.1), one observes that (4.1) is not supposed to be the correct definition for all pairs of disjoint morphisms, but only for those pairs $f=\left(f_{1}, f_{2}\right): \underline{M} \rightarrow N$ where $f_{1}\left(M_{1}\right) \subseteq N$ is "later" (in a suitable sense) than $f_{2}\left(\overline{\left.M_{2}\right)} \subseteq N\right.$. This would solve the 
problem concerning the equivariance property discussed above. The following definition formalizes a concept of time-ordering that allows us to prove our desired statements.

Definition 4.1. (a) Let $M \in$ Loc. A tuple $\left(U_{1}, \ldots, U_{n}\right)$ of causally convex open subsets $U_{i} \subseteq M$ is called time-ordered if $J_{M}^{+}\left(U_{i}\right) \cap U_{j}=\emptyset$, for all $i<j$.

(b) A tuple of pairwise disjoint morphisms $f=\left(f_{1}, \ldots, f_{n}\right): \underline{M} \rightarrow N$ is called time-ordered if the tuple $\left(f_{1}\left(M_{1}\right), \ldots, \overline{f_{n}}\left(M_{n}\right)\right)$ of causally convex open subsets $f_{i}\left(M_{i}\right) \subseteq N$ is time-ordered.

(c) A tuple of pairwise disjoint morphisms $f=\left(f_{1}, \ldots, f_{n}\right): \underline{M} \rightarrow N$ is called time-orderable if there exists a permutation $\rho \in \Sigma_{n}$ such that the tuple $f \rho=$ $\left(f_{\rho(1)}, \ldots, f_{\rho(n)}\right): \underline{M} \rho \rightarrow N$ is time-ordered. We call $\rho$ a time-ordering permutation for $f$ and note that time-ordering permutations are not necessarily unique.

Remark 4.2. By convention, all empty tuples $\emptyset \rightarrow N$ and all 1-tuples $f: M \rightarrow N$ are time-ordered. However, we would like to stress that for $n \geq 2$ not every tuple of pairwise disjoint morphisms $f: \underline{M} \rightarrow N$ is time-orderable. For example, consider $n=2$ and $f=\left(f_{1}, f_{2}\right): \underline{M} \rightarrow \bar{N}$ the inclusion of the following causally convex open subsets into the Lorentzian cylinder $N$ :

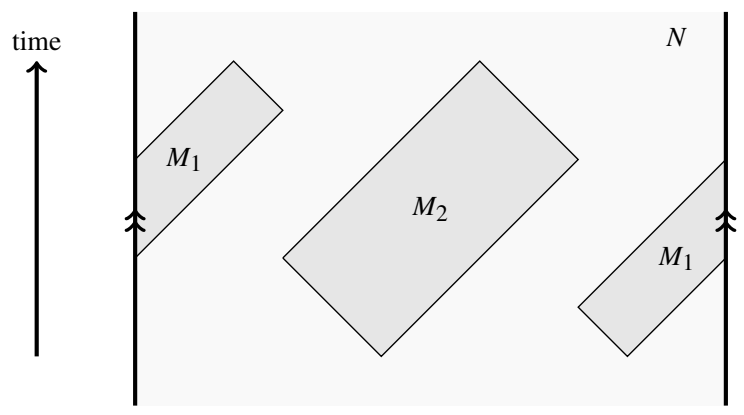

In this picture the left and right boundaries are identified as indicated, thereby producing the Lorentzian cylinder $N=\left(\mathbb{R} \times \mathbb{S}^{1}, g=-\mathrm{d} t^{2}+\mathrm{d} \phi^{2}, \mathfrak{t}=\frac{\partial}{\partial t}\right)$.

The following technical lemma is the crucial ingredient for our proofs below. We shall use the same notation and conventions for permutation group actions as in [Yau16].

Lemma 4.3. (i) Let $\rho \in \Sigma_{n}$ be a time-ordering permutation for the tuple of pairwise disjoint morphisms $\underline{f}=\left(f_{1}, \ldots, f_{n}\right): \underline{M} \rightarrow N$ and $\sigma \in \Sigma_{n}$ a permutation. Then $\sigma^{-1} \rho \in \Sigma_{n}$ is a time-ordering permutation for $f \sigma=\left(f_{\sigma(1)}, \ldots, f_{\sigma(n)}\right): \underline{M} \sigma \rightarrow N$. (ii) Let $\rho_{0} \in \Sigma_{n}$ be a time-ordering permutation for $f=\left(f_{1}, \ldots, f_{n}\right): \underline{M} \rightarrow N$ and $\rho_{i} \in \Sigma_{k_{i}}$ a time-ordering permutation for $\underline{g}_{i}=\left(g_{i 1}, \ldots, g_{i k_{i}}\right): \underline{L}_{i} \rightarrow M_{i}$, for $i=1, \ldots, n$. Then the permutation

$$
\rho_{0}\left\langle k_{1}, \ldots, k_{n}\right\rangle\left(\rho_{\rho_{0}(1)} \oplus \ldots \oplus \rho_{\rho_{0}(n)}\right) \in \Sigma_{k_{1}+\cdots+k_{n}},
$$

where $\rho_{0}\left\langle k_{1}, \ldots, k_{n}\right\rangle$ denotes the block permutation corresponding to $\rho_{0}$ and $\rho_{\rho_{0}(1)} \oplus$ $\ldots \oplus \rho_{\rho_{0}(n)}$ the sum permutation of the $\rho_{\rho_{0}(i)}$, is a time-ordering permutation for

$$
\underline{f}\left(\underline{g}_{1}, \ldots, \underline{g}_{n}\right):=\left(f_{1} g_{11}, \ldots, f_{n} g_{n k_{n}}\right):\left(\underline{L}_{1}, \ldots, \underline{L}_{n}\right) \longrightarrow N .
$$


(iii) Let $\underline{f}: \underline{M} \rightarrow N$ be a time-orderable tuple of pairwise disjoint morphisms and $\rho, \rho^{\prime} \in \Sigma_{n}$ time-ordering permutations for $f$. Then the right permutation $\rho^{-1} \rho^{\prime}$ : $\underline{f} \rho \rightarrow \underline{f} \rho^{\prime}$ is generated by transpositions of adjacent causally disjoint pairs of morphisms.

Proof. (i) Trivial.

(ii) Since

$$
\begin{aligned}
\underline{f} & \left(\underline{g}_{1}, \ldots, \underline{g}_{n}\right) \rho_{0}\left\langle k_{1}, \ldots, k_{n}\right\rangle\left(\rho_{\rho_{0}(1)} \oplus \ldots \oplus \rho_{\rho_{0}(n)}\right) \\
& =\left(\underline{f}_{\rho_{0}}\right)\left(\underline{g}_{\rho_{0}(1)} \rho_{\rho_{0}(1)}, \ldots, \underline{g}_{\rho_{0}(n)} \rho_{\rho_{0}(n)}\right),
\end{aligned}
$$

it is sufficient to prove that the composition of time-ordered tuples of pairwise disjoint morphisms is time-ordered. Therefore, assuming that $\frac{f}{f}$ and $\underline{g}_{i}$, for $i=1, \ldots, n$, are time-ordered, we have to show that $\left(f_{1} g_{11}, \ldots, \overline{f_{n}} g_{n k_{n}}\right)$ is time-ordered, i.e. $J_{N}^{+}\left(f_{i} g_{i i^{\prime}}\left(L_{i i^{\prime}}\right)\right) \cap f_{j} g_{j j^{\prime}}\left(L_{j j^{\prime}}\right)=\emptyset$ for the following two cases: Case 1 is $i<j$ and arbitrary $i^{\prime}=1, \ldots, k_{i}$ and $j^{\prime}=1, \ldots, k_{j}$. Case 2 is $i=j$ and $j<j^{\prime}$. Case 1 follows immediately from the hypothesis that $\underline{f}$ is time-ordered, i.e. $J_{N}^{+}\left(f_{i}\left(M_{i}\right)\right) \cap f_{j}\left(M_{j}\right)=$ $\emptyset$ for all $i<j$. For case 2 we use that $\underline{g}_{i}$ is time-ordered, i.e. $J_{M_{i}}^{+}\left(g_{i i^{\prime}}\left(L_{i i^{\prime}}\right)\right) \cap$ $g_{i j^{\prime}}\left(L_{i j^{\prime}}\right)=\emptyset$ for all $j<j^{\prime}$, and hence by the properties of Loc-morphisms

$$
J_{N}^{+}\left(f_{i} g_{i i^{\prime}}\left(L_{i i^{\prime}}\right)\right) \cap f_{i} g_{i j^{\prime}}\left(L_{i j^{\prime}}\right)=f_{i}\left(J_{M_{i}}^{+}\left(g_{i i^{\prime}}\left(L_{i i^{\prime}}\right)\right) \cap g_{i j^{\prime}}\left(L_{i j^{\prime}}\right)\right)=\emptyset .
$$

This proves that $\left(f_{1} g_{11}, \ldots, f_{n} g_{n k_{n}}\right)$ is time-ordered.

(iii) Suppose that $\rho^{-1} \rho^{\prime}: \underline{f} \rho \rightarrow \underline{f} \rho^{\prime}$ reverses the time-ordering between $f_{k}$ and $f_{\ell}$, i.e. $\rho(i)=k=\rho^{\prime}\left(i^{\prime}\right)$ and $\rho(j)=\ell=\rho^{\prime}\left(j^{\prime}\right)$ with $i<j$ and $j^{\prime}<i^{\prime}$ or vice versa with $j<i$ and $i^{\prime}<j^{\prime}$. Let us consider the case $i<j$ and $j^{\prime}<i^{\prime}$, the other one being similar. By hypothesis, we have that $J_{N}^{+}\left(f_{\rho(i)}\left(M_{\rho(i)}\right)\right) \cap f_{\rho(j)}\left(M_{\rho(j)}\right)=\varnothing$ and $J_{N}^{+}\left(f_{\rho^{\prime}\left(j^{\prime}\right)}\left(M_{\rho^{\prime}\left(j^{\prime}\right)}\right)\right) \cap f_{\rho^{\prime}\left(i^{\prime}\right)}\left(M_{\rho^{\prime}\left(i^{\prime}\right)}\right)=\emptyset$, which is equivalent to $f_{k} \perp f_{\ell}$ being causally disjoint. Summing up, this proves that every pair $\left(f_{k}, f_{\ell}\right)$ of morphisms whose time-ordering is reversed by $\rho^{-1} \rho^{\prime}$ is causally disjoint $f_{k} \perp f_{\ell}$.

To conclude the proof, let us recall that every permutation $\sigma:\left(h_{1}, \ldots, h_{n}\right) \rightarrow$ $\left(h_{\sigma(1)}, \ldots, h_{\sigma(n)}\right)$ admits a (not necessarily unique) factorization into adjacent transpositions that flip only elements whose order is reversed by $\sigma$. (One way to obtain such a factorization is as follows: Start from $\left(h_{1}, \ldots, h_{n}\right)$ and move by adjacent transpositions the element $h_{\sigma(1)}$ to the leftmost position. Then move by adjacent transpositions the element $h_{\sigma(2)}$ to the second leftmost position, and so on.) This implies that we obtain a factorization $\rho^{-1} \rho^{\prime}=\tau_{1} \cdots \tau_{N}: \underline{f} \rho \rightarrow \underline{f} \rho^{\prime}$, where each $\tau_{l}: \underline{f} \rho \tau_{1} \cdots \tau_{l-1} \rightarrow \underline{f} \rho \tau_{1} \cdots \tau_{l}$ transposes two adjacent Loc-morphisms whose time-ordering is reversed by $\rho^{-1} \rho^{\prime}$. Our result in the previous paragraph then implies that each $\tau_{l}$ is a transposition of adjacent causally disjoint pairs of morphisms, which completes our proof.

Lemma 4.3 plays a crucial role in the following definition of time-orderable prefactorization algebras because it ensures that time-orderable tuples of pairwise disjoint morphisms are composable and carry permutation actions. A time-orderable prefactorization algebra $\mathfrak{F}$ on Loc with values in $\mathbf{C}$ is given by the following data:

(i) for each $M \in \mathbf{L o c}$, an object $\mathfrak{F}(M) \in \mathbf{C}$; 
(ii) for each time-orderable tuple $\underline{f}=\left(f_{1}, \ldots, f_{n}\right): \underline{M} \rightarrow N$ of pairwise disjoint morphisms, a C-morphism $\mathfrak{F}(f): \bigotimes_{i=1}^{n} \mathfrak{F}\left(M_{i}\right) \rightarrow \mathfrak{F}(N)$ (called time-ordered product), with the convention that to the empty tuple $\emptyset \rightarrow N$ is assigned a morphism $I \rightarrow \mathfrak{F}(N)$ from the monoidal unit.

These data are required to satisfy the analogs of the prefactorization algebra axioms from Sect. 2.2 for time-orderable tuples. A morphism $\zeta: \mathfrak{F} \rightarrow \mathfrak{G}$ of time-orderable prefactorization algebras is a family $\zeta_{M}: \mathfrak{F}(M) \rightarrow \mathfrak{G}(M)$ of C-morphisms, for all $M \in \mathbf{L o c}$, that is compatible with the time-ordered products as in (2.3).

Definition 4.4. We denote by tPFA the category of time-orderable prefactorization algebras on Loc. In analogy to Definitions 2.11 and 2.14, we introduce the full subcategories $\mathbf{t P F A}^{\text {add }}, \mathbf{t P F A}^{c}, \mathbf{t P F A}^{\text {add }, c} \subseteq \mathbf{\text { tPFA }}$ of additive, Cauchy constant and Cauchy constant additive time-orderable prefactorization algebras.

Remark 4.5. Each ordinary prefactorization algebra on Loc defines a time-orderable one by restriction to time-orderable tuples of pairwise disjoint morphisms. This defines a functor PFA $\rightarrow$ tPFA, which is faithful, but not necessarily full due to the fact that not all pairwise disjoint tuples $f: \underline{M} \rightarrow N$ are time-orderable, cf. Remark 4.2. This functor clearly preserves both additivity and Cauchy constancy.

With these preparations we can now carry out our envisaged construction of a timeorderable prefactorization algebra $\mathbb{F}[\mathfrak{A}] \in$ tPFA from a given algebraic quantum field theory $\mathfrak{A} \in \mathbf{A Q F T}$. In particular, we can now complete our attempt from the beginning of this section to define the time-ordered factorization products. Let $f=\left(f_{1}, \ldots, f_{n}\right)$ : $\underline{M} \rightarrow N$ be a time-orderable tuple of pairwise disjoint morphisms with time-ordering permutation $\rho \in \Sigma_{n}$. We define the corresponding time-ordered product $\mathbb{F}[\mathfrak{A}](f)$ : $\bigotimes_{i=1}^{n} \mathfrak{A}\left(M_{i}\right) \rightarrow \mathfrak{A}(N)$ by the commutative diagram

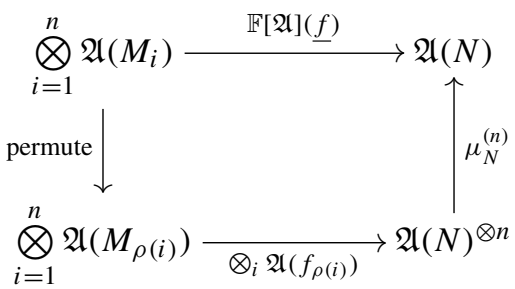

in $\mathbf{C}$, where $\mu_{N}^{(n)}$ denotes the $n$-ary multiplication in the associative and unital algebra $\mathfrak{A}(N)$ in the given order, i.e. $\mu_{N}^{(n)}\left(a_{1} \otimes \cdots \otimes a_{n}\right)=a_{1} \cdots a_{n}$ with juxtaposition denoting multiplication in $\mathfrak{A}(N)$. As before, for $n=0$ we assign to the empty tuple $\emptyset \rightarrow N$ the C-morphism $\eta_{N}: I \rightarrow \mathfrak{A}(N)$ corresponding to the unit of $\mathfrak{A}(N)$.

Lemma 4.6. The C-morphism $\mathbb{F}[\mathfrak{A}](f): \bigotimes_{i=1}^{n} \mathfrak{A}\left(M_{i}\right) \rightarrow \mathfrak{A}(N)$ defined in (4.7) does not depend on the choice of time-ordering permutation for $\underline{f}: \underline{M} \rightarrow N$.

Proof. Consider time-ordering permutations $\rho, \rho^{\prime} \in \Sigma_{n}$ for $\underline{f}$. Recalling Lemma 4.3 (iii), the right permutation $\rho^{-1} \rho^{\prime}: f \rho \rightarrow f \rho^{\prime}$ is generated by transpositions of adjacent causally disjoint pairs of morphisms. Hence, the claim follows from the Einstein causality axiom (2.7) of the algebraic quantum field theory $\mathfrak{A} \in$ AQFT.

Theorem 4.7. Let $\mathfrak{A} \in \mathbf{A Q F T}$ be an algebraic quantum field theory. Then the following data defines a time-orderable prefactorization algebra $\mathbb{F}[\mathfrak{A}] \in$ tPFA: 
(i) for each $M \in \mathbf{L o c}$, define $\mathbb{F}[\mathfrak{A}](M):=\mathfrak{A}(M) \in \mathbf{C}$ via the forgetful functor $\mathbf{A l g} \rightarrow$ C;

(ii) for each time-orderable tuple of pairwise disjoint morphisms $f=\left(f_{1}, \ldots, f_{n}\right)$ : $\underline{M} \rightarrow N$, define the time-ordered product $\mathbb{F}[\mathfrak{A}](f): \bigotimes_{i=1}^{n} \mathbb{F}[\overline{\mathfrak{A}}]\left(M_{i}\right) \rightarrow \mathbb{F}[\mathfrak{A}](N)$

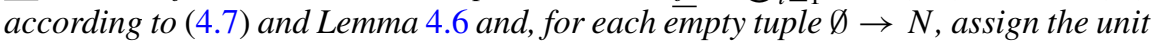
$\eta_{N}: I \rightarrow \mathbb{F}[\mathfrak{A}](N)$ of $\mathfrak{A}(N)$.

The assignment $\mathfrak{A} \mapsto \mathbb{F}[\mathfrak{A}]$ canonically extends to a functor $\mathbb{F}:$ AQFT $\rightarrow$ tPFA.

Proof. Lemma 4.3 immediately implies that $\mathbb{F}[\mathfrak{A}]$ satisfies the axioms of time-orderable prefactorization algebras. More explicitly, Lemma 4.3 (i) implies the equivariance axiom (2.2) for all time-orderable tuples and Lemma 4.3 (ii) implies the composition axiom (2.1) for all time-orderable tuples. By definition, we also have that $\mathbb{F}[\mathfrak{A}]\left(\mathrm{id}_{M}\right)=$ $\operatorname{id}_{\mathbb{F}[\mathfrak{A}](M)}$, for all $M \in$ Loc.

Concerning functoriality of the assignment $\mathfrak{A} \mapsto \mathbb{F}[\mathfrak{A}]$, we have to show that every AQFT-morphism $\kappa: \mathfrak{A} \rightarrow \mathfrak{B}$ canonically defines a tPFA-morphism $\mathbb{F}[\kappa]: \mathbb{F}[\mathfrak{A}] \rightarrow$ $\mathbb{F}[\mathfrak{B}]$. Observe that, for every time-orderable tuple $\underline{f}: \underline{M} \rightarrow N$ with time-ordering permutation $\rho \in \Sigma_{n}$, the diagram

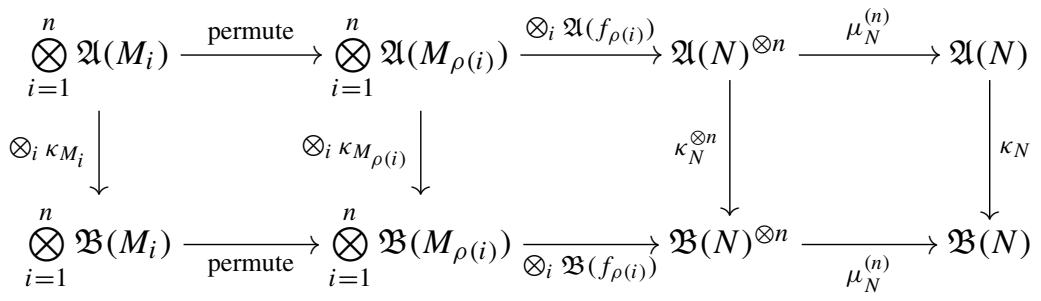

in $\mathbf{C}$ commutes. Hence, the family $\kappa_{M}: \mathfrak{A}(M) \rightarrow \mathfrak{B}(M)$ of $\mathbf{C}$-morphisms defines a tPFA-morphism $\mathbb{F}[\kappa]: \mathbb{F}[\mathfrak{A}] \rightarrow \mathbb{F}[\mathfrak{B}]$.

Proposition 4.8. $\mathfrak{A} \in \mathbf{A Q F T}$ is additive (respectively Cauchy constant) if and only if $\mathbb{F}[\mathfrak{A}] \in$ tPFA is additive (respectively Cauchy constant). In particular, the functor $\mathbb{F}:$ AQFT $\rightarrow$ tPFA from Theorem 4.7 restricts to full subcategories as $\mathbb{F}:$ AQFT ${ }^{\text {add }} \rightarrow$ tPFA $^{\text {add }}, \mathbb{F}: \mathbf{A Q F T}^{c} \rightarrow$ tPFA $^{c}$ and $\mathbb{F}: \mathbf{A Q F T}^{\text {add }, c} \rightarrow$ tPFA $^{\text {add }, c}$.

Proof. Let us recall that, by our construction, the underlying functors $\mathbb{F}[\mathfrak{A}]=\mathfrak{A}$ : Loc $\rightarrow \mathbf{C}$ to the category $\mathbf{C}$ coincide. It is then a consequence of Remark 2.17 that $\mathbb{F}[\mathfrak{A}]$ is additive if and only if $\mathfrak{A}$ is additive. Furthermore, because the forgetful functor $\mathbf{A l g} \rightarrow \mathbf{C}$ preserves and detects isomorphisms, it follows that $\mathbb{F}[\mathfrak{A}]$ is Cauchy constant if and only if $\mathfrak{A}$ is Cauchy constant.

\section{Equivalence Theorem}

5.1. Main result. The aim of this section is to prove that our two constructions from Sects. 3 and 4 are inverse to each other when restricted to their common domain of validity. Recall that in Sect. 3 we considered Cauchy constant additive prefactorization algebras and constructed a functor $\mathbb{A}: \mathbf{P F A}^{\text {add }, c} \rightarrow \mathbf{A Q F T}^{\text {add }, c}$ to the category of Cauchy constant additive algebraic quantum field theories, cf. Theorem 3.11. Because the construction presented in Sect. 3 only involves time-orderable tuples of disjoint morphisms, this functor factors through the forgetful functor $\mathbf{P F A}^{\text {add, } c} \rightarrow \mathbf{t P F A}^{\text {add }, c}$ (cf. 
Remark 4.5) to the category of Cauchy constant additive time-orderable prefactorization algebras, cf. Definition 4.4. We shall denote the resulting functor by the same symbol $\mathbb{A}$ : $\mathbf{t P F A}^{\text {add }, c} \rightarrow \mathbf{A Q F T}^{\text {add }, c}$. Let us further recall the functor $\mathbb{F}: \mathbf{A Q F T}^{\text {add }, c} \rightarrow \mathbf{t P F A}^{\text {add }, c}$ from Theorem 4.7 and Proposition 4.8. Our main result is the following equivalence theorem.

Theorem 5.1. The two functors $\mathbb{A}: \mathbf{t P F A}^{\text {add }, c} \rightarrow \mathbf{A Q F T}^{\mathrm{add}, c}$ and $\mathbb{F}: \mathbf{A Q F T}^{\text {add }, c} \rightarrow$ tPFA $^{\text {add, } c}$ are inverses of each other. As a consequence, the category AQFT ${ }^{\text {add }, c}$ of Cauchy constant additive algebraic quantum field theories is isomorphic to the category tPFA $^{\text {add, } c}$ of Cauchy constant additive time-orderable prefactorization algebras.

Proof. The only non-trivial check to confirm that $\mathbb{A} \circ \mathbb{F}=\mathrm{id}_{\mathbf{A Q F T}}{ }^{\text {add }, c}$ amounts to show that, for every $\mathfrak{A} \in \mathbf{A Q F T}{ }^{\text {add, } c}$, the multiplications on $\mathbb{A}[\mathbb{F}[\mathfrak{A}]](M)$ and on $\mathfrak{A}(M)$ coincide, for all $M \in$ Loc. By (3.1) and (4.7), the multiplication on $\mathbb{A}[\mathbb{F}[\mathfrak{A}]](M)$ is given by

$$
\mathfrak{A}(M) \otimes \mathfrak{A}(M) \stackrel{\mathfrak{A}\left(\iota_{U_{+}}^{M}\right) \otimes \mathfrak{A}\left(\iota_{U_{-}}^{M}\right)}{\cong} \mathfrak{A}\left(U_{+}\right) \otimes \mathfrak{A}\left(U_{-}\right) \stackrel{\mathfrak{A}\left(\iota_{U_{+}}^{M}\right) \otimes \mathfrak{A}\left(\iota_{U_{-}}^{M}\right)}{\longrightarrow} \mathfrak{A}(M)^{\otimes 2} \stackrel{\mu_{M}}{\longrightarrow} \mathfrak{A}(M),
$$

where $\iota_{\underline{U}}^{M}=\left(\iota_{U_{+}}^{M}, \iota_{U_{-}}^{M}\right): \underline{U} \rightarrow M$ is any object of $\mathbf{P}_{M}$. This clearly coincides with the original multiplication $\mu_{M}$ on $\mathfrak{A}(M)$.

Conversely, to show that $\mathbb{F} \circ \mathbb{A}=\mathrm{id}_{\mathbf{t P F A}^{\text {add }, c}}$, we have to confirm that the time-ordered products of $\mathbb{F}[\mathbb{A}[\mathfrak{F}]] \in \mathbf{t P F A}^{\text {add }, c}$ coincide with the original time-ordered products of $\mathfrak{F} \in$ tPFA $^{\text {add, } c}$. In arity $n=0$ and $n=1$ this is obvious. For $n \geq 2$, this is more complicated and requires some preparations. Using equivariance under permutation actions, it is sufficient to compare the time-ordered products for time-ordered (in contrast to timeorderable) tuples $f=\left(f_{1}, \ldots, f_{n}\right): \underline{M} \rightarrow N$. Because of additivity, we can further restrict to the case where $\underline{f}: \underline{M} \rightarrow N$ has relatively compact images, i.e. $f_{i}\left(M_{i}\right) \subseteq N$ is relatively compact, for all $i=1, \ldots, n$. We shall now show that, due to Cauchy constancy, we can further restrict our attention to time-ordered tuples $\underline{h}=\left(h_{1}, \ldots, h_{n}\right)$ : $\underline{L} \rightarrow N$ with relatively compact images for which there exists a Cauchy surface $\Sigma$ of $N$ such that

$h_{1}\left(L_{1}\right), \ldots, h_{n-1}\left(L_{n-1}\right) \subseteq \Sigma_{+}:=I_{N}^{+}(\Sigma) \subseteq N$ and $h_{n}\left(L_{n}\right) \subseteq \Sigma_{-}:=I_{N}^{-}(\Sigma) \subseteq N$.

Indeed, given any time-ordered tuple $\underline{f}: \underline{M} \rightarrow N$ with relatively compact images, we shall prove below that there exists a family of Cauchy morphisms $g_{i}: L_{i} \stackrel{c}{\rightarrow} M_{i}$, for $i=1, \ldots, n$, such that $\underline{h}:=f\left(g_{1}, \ldots, g_{n}\right)=\left(f_{1} g_{1}, \ldots, f_{n} g_{n}\right): \underline{L} \rightarrow N$ admits a Cauchy surface $\Sigma$ that satisfies (5.2). Cauchy constancy and the fact that the time-ordered products of $\mathbb{F}[\mathbb{A}[\mathfrak{F}]]$ and $\mathfrak{F}$ agree in arity $n=1$ then implies that $\mathbb{F}[\mathbb{A}[\mathfrak{F}]](f)=\mathfrak{F}(f)$ if and only if $\mathbb{F}[\mathbb{A}[\mathfrak{F}]](\underline{h})=\mathfrak{F}(\underline{h})$. To exhibit such a family of Cauchy morphisms for $\underline{f}: \underline{M} \rightarrow N$, let us choose Cauchy surfaces $\Sigma_{i}$ of $M_{i}$, for $i=1, \ldots, n$, and define $L_{i}:=I_{M_{i}}^{+}\left(\Sigma_{i}\right)$, for $i=1, \ldots, n-1$, and $L_{n}:=I_{M_{n}}^{-}\left(\Sigma_{n}\right)$. Let us further define $g_{i}:=\iota_{L_{i}}^{M_{i}}: L_{i} \stackrel{c}{\rightarrow} M_{i}$ by subset inclusion, for $i=1, \ldots, n$. A Cauchy surface $\Sigma$ of $N$ is constructed by extending via [BS06, Theorem 3.8] the compact and achronal subset

$$
\widetilde{\Sigma}:=\bigcup_{i=1}^{n}\left(\overline{f_{i}\left(\Sigma_{i}\right)} \backslash I_{N}^{+}\left(\bigcup_{j=i+1}^{n} \overline{f_{j}\left(\Sigma_{j}\right)}\right)\right) \subseteq N
$$


By direct inspection one observes that $\Sigma$ fulfills (5.2).

Using (5.2), we obtain a factorization

$$
\underline{h}=\iota_{\underline{\Sigma}}^{N}\left(\left(h_{1}^{\Sigma_{+}}, \ldots, h_{n-1}^{\Sigma_{+}}\right), h_{n}^{\Sigma_{-}}\right),
$$

where on the right-hand side we regard $h_{i}^{\Sigma_{+}}: L_{i} \rightarrow \Sigma_{+}$as morphisms to $\Sigma_{+}$, for $i=$ $1, \ldots, n-1$, and $h_{n}^{\Sigma_{-}}: L_{n} \rightarrow \Sigma_{-}$as a morphism to $\Sigma_{-}$. Iterating this construction, we observe that it is sufficient to prove that $\mathbb{F}[\mathbb{A}[\mathfrak{F}]]\left(\iota_{\Sigma}^{N}\right)=\mathfrak{F}\left(\iota_{\Sigma}^{N}\right)$, for all $\iota_{\Sigma}^{N}=\left(\iota_{\Sigma_{+}}^{N}, \iota_{\Sigma_{-}}^{N}\right)$ : $\underline{\Sigma} \rightarrow N$, where $N \in$ Loc and the Cauchy surface $\Sigma$ of $N$ is arbitrary. Using (4.7) and (3.1), we obtain that $\mathbb{F}[\mathbb{A}[\mathfrak{F}]]\left(l_{\underline{\Sigma}}^{N}\right): \mathfrak{F}\left(\Sigma_{+}\right) \otimes \mathfrak{F}\left(\Sigma_{-}\right) \rightarrow \mathfrak{F}(N)$ is given by

$$
\mathfrak{F}\left(\Sigma_{+}\right) \otimes \mathfrak{F}\left(\Sigma_{-}\right) \stackrel{\mathfrak{F}\left(l_{\Sigma_{+}}^{N}\right) \otimes \mathfrak{F}\left(l_{\Sigma_{-}}^{N}\right)}{\longrightarrow} \mathfrak{F}(N) \otimes \mathfrak{F}(N) \stackrel{\mathfrak{F}\left(\iota_{\Sigma_{+}}^{N}\right) \otimes \mathfrak{F}\left(\iota_{\Sigma_{-}}^{N}\right)}{\cong} \mathfrak{F}\left(\Sigma_{+}\right) \otimes \mathfrak{F}\left(\Sigma_{-}\right) \stackrel{\mathfrak{F}\left(\iota_{\underline{\Sigma}}^{N}\right)}{\longrightarrow} \mathfrak{F}(N)
$$

which clearly coincides with the original time-ordered product $\mathfrak{F}\left(\iota_{\underline{\Sigma}}^{N}\right): \mathfrak{F}\left(\Sigma_{+}\right) \otimes \mathfrak{F}\left(\Sigma_{-}\right) \rightarrow$ $\mathfrak{F}(N)$. This concludes our proof.

Remark 5.2. We would like to mention very briefly a more abstract operadic perspective on the Equivalence Theorem 5.1. Recall from [BSW17] that there exists a Set-valued colored operad $\mathcal{O}_{(\mathbf{L o c}, \perp)}$ whose category of $\mathbf{C}$-valued algebras is the category of algebraic quantum field theories, i.e. $\mathbf{A Q F T}=\mathbf{A l g}_{\mathcal{O}_{(\operatorname{Loc}, \perp)}}(\mathbf{C})$. We can also define a Set-valued colored operad $\mathcal{P}_{\text {Loc }}$ such that tPFA $=\mathbf{A} \mathbf{A g}_{\mathcal{P}_{\text {Loc }}}(\mathbf{C})$. Concretely, the colors of $\mathcal{P}_{\text {Loc }}$ are the objects of Loc and the sets of operations are $\mathcal{P}_{\mathbf{L o c}}(\underline{M}):=\{$ all time-orderable tuples $\underline{f}$ : $\underline{M} \rightarrow N\}$. Operadic composition is given by (4.4), the operadic units $\operatorname{are~}_{M} \in \mathcal{P}_{\text {Loc }}\left(\begin{array}{l}M \\ M\end{array}\right)$ and the permutation actions are $\mathcal{P}_{\mathbf{L o c}}(\sigma): \mathcal{P}_{\mathbf{L o c}}(\underline{M}) \rightarrow \mathcal{P}_{\mathbf{L o c}}(\underline{M} \sigma), \underline{f} \mapsto \underline{f \sigma}$, for $\sigma \in \Sigma_{n}$. Using Lemma 4.3 and the definition of the colored operad $\mathcal{O}_{(\mathbf{L o c}, \perp)} \overline{\text { given in }}$ [BSW17], one immediately observes that the component maps

$$
\Phi: \mathcal{P}_{\mathbf{L o c}}(\underline{\underline{M}}) \longrightarrow \mathcal{O}_{(\mathbf{L o c}, \perp)}(\underline{\underline{M}}), \quad \underline{f} \longmapsto\left[\rho^{-1}, \underline{f}\right]
$$

define a colored operad morphism $\Phi: \mathcal{P}_{\text {Loc }} \rightarrow \mathcal{O}_{(\text {Loc }, \perp)}$, where $\rho \in \Sigma_{n}$ is any timeordering permutation for $\underline{f}$. The associated pullback functor $\Phi^{*}: \mathbf{A l g}_{\mathcal{O}_{(\mathbf{L o c}, \perp)}}(\mathbf{C}) \rightarrow$ $\operatorname{Alg}_{\mathcal{P}_{\text {Loc }}}(\mathbf{C})$ is then precisely our functor $\mathbb{F}:$ AQFT $\rightarrow$ tPFA from Theorem 4.7. By operadic left Kan extension, there exists an adjunction

$$
\Phi_{!}: \text {tPFA } \rightleftarrows \text { AQFT }: \Phi^{*}=\mathbb{F}
$$

Theorem 5.1 then states that restricting both sides of this adjunction to Cauchy constant and additive theories induces an adjoint equivalence $\mathbb{A}: \mathbf{t P F A}^{\text {add }, c} \stackrel{\sim}{\rightleftarrows} \mathbf{A Q F T}^{\text {add }, c}: \mathbb{F}$.

We expect that this operadic perspective will become important when considering the case where the target category $\mathbf{C}$ is a higher category or model category. This generalization is crucial for the description of quantum gauge theories in terms of factorization algebras [CG17] or algebraic quantum field theories [BSS15, BS17, BSW19b, BS19]. The adjunction (5.7) then becomes a Quillen adjunction between model categories, and a reasonable equivalence theorem would state that suitable restrictions to homotopy-invariant analogs of Cauchy constant and additive theories induce a Quillen equivalence. Proving such an equivalence theorem in a higher categorical context is technically complicated and will not be considered in the present paper. 
5.2. Transfer of $*$-involutions. Algebraic quantum field theories are typically endowed with the structure of a $*$-involution, i.e. they assign $*$-algebras to spacetimes. The aim of this subsection is to introduce $*$-involutions for Cauchy constant additive time-orderable prefactorization algebras by transferring via our Equivalence Theorem 5.1 the usual concept of $*$-involution for algebraic quantum field theories. The formalization of $*-$ structures requires the underlying category $\mathbf{C}$ to be an involutive category, see e.g. [BSW19a]. To simplify our presentation, we consider only the most relevant case where $\mathbf{C}=\mathbf{V e c}_{\mathbb{C}}$ is the symmetric monoidal category of complex vector spaces, endowed with the usual involution functor $\overline{(-)}: \mathbf{V e c}_{\mathbb{C}} \rightarrow \mathbf{V e c}_{\mathbb{C}}$ that assigns to a complex vector space $V \in \mathbf{V e c}_{\mathbb{C}}$ its complex conjugate vector space $\bar{V} \in \mathbf{V e c}_{\mathbb{C}}$. The complex conjugate of a $\mathbb{C}$-linear map $L: V \rightarrow W$ is denoted by $\bar{L}: \bar{V} \rightarrow \bar{W}$. We note that $\overline{\bar{V}}=V$, for all $V \in$ $\mathbf{V e c}_{\mathbb{C}}$, and that $\bar{V} \otimes \bar{W}=\overline{V \otimes W}$, for all $V, W \in \mathbf{V e c}_{\mathbb{C}}$. Moreover, complex conjugation on $\mathbb{C}$ defines a $\mathbb{C}$-linear map $*: \mathbb{C} \rightarrow \overline{\mathbb{C}}$ that satisfies $\bar{*} \circ * \mathrm{id}_{\mathbb{C}}: \mathbb{C} \rightarrow \overline{\overline{\mathbb{C}}}=\mathbb{C}$.

The results in [BSW19a] allow us to endow the category AQFT of $\mathbf{V e c}_{\mathbb{C}}$-valued algebraic quantum field theories with an involutive structure, which we denote with an abuse of notation also by $\overline{(-)}:$ AQFT $\rightarrow$ AQFT. Concretely, the complex conjugate $\overline{\mathfrak{A}} \in \mathbf{A Q F T}$ of $\mathfrak{A} \in \mathbf{A Q F T}$ is determined by the functor $\overline{\mathfrak{A}}:$ Loc $\rightarrow$ Alg that assigns to $M \in$ Loc the algebra $\overline{\mathfrak{A}}(M)$ whose underlying vector space is $\overline{\mathfrak{A}(M)}$ and whose multiplication and unit are $\overline{\mu_{M}^{\mathrm{op}}}: \overline{\mathfrak{A}(M)} \otimes \overline{\mathfrak{A}(M)}=\overline{\mathfrak{A}(M) \otimes \mathfrak{A}(M)} \rightarrow \overline{\mathfrak{A}(M)}$ and $\overline{\eta_{M}} \circ *: \mathbb{C} \rightarrow \overline{\mathbb{C}} \rightarrow \overline{\mathfrak{A}(M)}$. (The opposite multiplication appears here because the relevant $*$-involutions on algebras are order-reversing, i.e. $(a b)^{*}=b^{*} a^{*}$.) To a Locmorphism $f: M \rightarrow N$, it assigns the algebra morphism determined by the complex conjugate $\mathbb{C}$-linear map $\overline{\mathfrak{A}}(f):=\overline{\mathfrak{A}(f)}: \overline{\overline{\mathfrak{A}}(M)} \rightarrow \overline{\mathfrak{A}(N)}$. We note that $\overline{\overline{\mathfrak{A}}}=\mathfrak{A}$, for all $\mathfrak{A} \in$ AQFT. A $*$-involution on an algebraic quantum field theory $\mathfrak{A} \in \mathbf{A Q F T}$ is then defined as an AQFT-morphism $* \mathfrak{A}: \mathfrak{A} \rightarrow \overline{\mathfrak{A}}$ that satisfies $\overline{* \mathfrak{A}} \circ * \mathfrak{A}=\operatorname{id}_{\mathfrak{A}}: \mathfrak{A} \rightarrow$ $\overline{\overline{\mathfrak{A}}}=\mathfrak{A}$. We denote by $*$ AQFT the category whose objects are pairs $(\mathfrak{A}, * \mathfrak{A})$ consisting of an $\mathfrak{A} \in \mathbf{A Q F T}$ and a $*$-involution $*_{\mathfrak{A}}$ and whose morphisms are AQFT-morphisms $\kappa: \mathfrak{A} \rightarrow \mathfrak{B}$ that preserve the $*$-involutions, i.e. $\bar{\kappa} \circ *_{\mathfrak{A}}=*_{\mathfrak{B}} \circ \kappa$. It is easy to confirm that our definition agrees with the usual one from the literature [BFV03,FV12,BDFY15] that considers functors Loc $\rightarrow * \mathbf{A l g}$ to the category of $*$-algebras over $\mathbb{C}$, see [BSW19a] for more details.

The involutive structure on AQFT restricts to an involution functor $\overline{(-)}: \mathbf{A Q F T}^{\text {add }, c} \rightarrow$ AQFT $^{\text {add }, c}$ on the full subcategory of Cauchy constant additive algebraic quantum field theories. By the Equivalence Theorem 5.1, we obtain a transferred involution functor $\overline{(-)}: \mathbf{t P F A}^{\text {add }, c} \rightarrow \mathbf{t P F A}^{\text {add }, c}$ on the category of Cauchy constant additive timeorderable prefactorization algebras, which we denote with an abuse of notation by the same symbol. Concretely, the complex conjugate $\overline{\mathfrak{F}} \in \mathbf{t P F A}^{\text {add }, c}$ of $\mathfrak{F} \in \mathbf{t P F A}^{\text {add }, c}$ is given by $\overline{\mathfrak{F}}:=\mathbb{F}[\overline{\mathbb{A}[\mathfrak{F}]}]$. A $*$-involution on a Cauchy constant additive time-orderable prefactorization algebra $\mathfrak{F} \in$ tPFA $^{\text {add }, c}$ is then defined as a tPFA ${ }^{\text {add }, c}$-morphism $* \mathfrak{F}:$ $\mathfrak{F} \rightarrow \overline{\mathfrak{F}}$ that satisfies $\overline{* \mathfrak{F}} \circ * \mathfrak{F}=\operatorname{id}_{\mathfrak{F}}: \mathfrak{F} \rightarrow \overline{\overline{\mathfrak{F}}}=\mathfrak{F}$. We denote by $*$ tPFA $^{\text {add }, c}$ the category whose objects are pairs $(\mathfrak{F}, * \mathfrak{F})$ consisting of a $\mathfrak{F} \in \mathbf{t P F A}^{\text {add }, c}$ and a $*$-involution $* \mathfrak{F}$ and whose morphisms are tPFA ${ }^{\text {add }, c}$-morphisms $\zeta: \mathfrak{F} \rightarrow \mathfrak{G}$ that preserve the $*$-involutions, i.e. $\bar{\zeta} \circ *_{\mathfrak{F}}=*_{\mathfrak{G}} \circ \zeta$. By construction, the Equivalence Theorem 5.1 determines an equivalence $* \mathbf{A Q F T} \mathbf{T}^{\text {add }, c} \simeq * \mathbf{t} \mathbf{P F A} \mathbf{A}^{\text {add }, c}$ between theories with $*$-involutions.

From our constructions above, it remains unclear if there exists an intrinsic definition of the complex conjugate prefactorization algebra $\overline{\mathfrak{F}}=\mathbb{F}[\overline{\mathbb{A}[\mathfrak{F}]}] \in \mathbf{t P F A}^{\text {add }, c}$ that does 
not rely on Cauchy constancy and additivity, i.e. that is applicable to all time-orderable prefactorization algebras in tPFA. Unfortunately, this does not seem to be the case. To understand and explain these issues, let us compute explicitly the complex conjugate factorization product $\overline{\mathfrak{F}}\left(\iota_{\left(\Sigma_{+}, \Sigma_{-}\right)}^{M}\right): \overline{\mathfrak{F}}\left(\Sigma_{+}\right) \otimes \overline{\mathfrak{F}}\left(\Sigma_{-}\right) \rightarrow \overline{\mathfrak{F}}(M)$ for the time-ordered pair of inclusions $\iota_{\Sigma_{ \pm}}^{M}: \Sigma_{ \pm} \rightarrow M$ determined by a choice of Cauchy surface $\Sigma \subset M$ via $\Sigma_{ \pm}=I_{M}^{ \pm}(\Sigma)$. Using (4.7) and (3.1), we obtain the commutative diagram

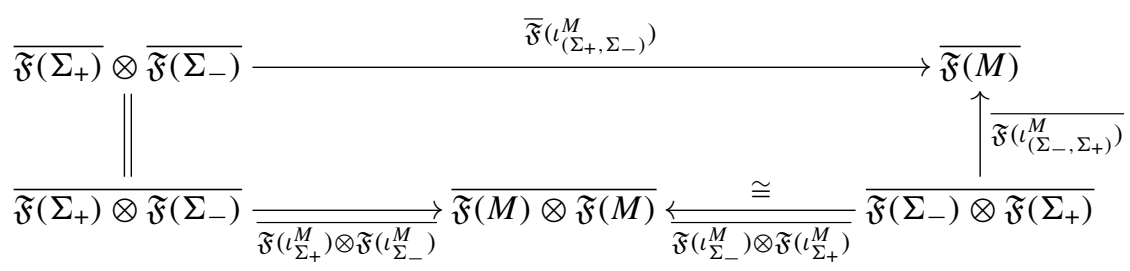

which relates the factorization product $\overline{\mathfrak{F}}\left(\iota_{\left(\Sigma_{+}, \Sigma_{-}\right)}^{M}\right)$ of $\overline{\mathfrak{F}}$ to the factorization product $\mathfrak{F}\left(\iota_{\left(\Sigma_{-}, \Sigma_{+}\right)}^{M}\right)$ of $\mathfrak{F}$. Note that the bottom horizontal arrow uses Cauchy constancy explicitly. Physically speaking, it propagates observables from the future region $\Sigma_{+}$to the past region $\Sigma_{-}$and observables from $\Sigma_{-}$to $\Sigma_{+}$. In particular, in absence of Cauchy constancy, the diagram in (5.8) can not be used to determine the factorization product $\overline{\mathfrak{F}}\left(\iota_{\left(\Sigma_{+}, \Sigma_{-}\right)}^{M}\right)$ from the factorization products of $\mathfrak{F}$, because the second bottom horizontal arrow is in general not invertible.

5.3. Example: the free Klein-Gordon field. We apply our general Equivalence Theorem 5.1 to the simple example given by the free Klein-Gordon field and thereby recover the results from [GR17]. Let us briefly recall the algebraic quantum field theory description of the free Klein-Gordon field. For every $M \in$ Loc, consider the Klein-Gordon operator $P_{M}:=-\square_{M}+m^{2}: C^{\infty}(M) \rightarrow C^{\infty}(M)$, where $\square_{M}$ is the d'Alembert operator and $m^{2} \geq 0$ is a mass parameter. $P_{M}$ admits a unique retarded/advanced Green's operator $G_{M}^{ \pm}: C_{\mathrm{c}}^{\infty}(M) \rightarrow C^{\infty}(M)$, where the subscript 'c' denotes compactly supported functions. The $\mathbb{R}$-vector space $\mathcal{V}(M)$ of linear observables on $M$ is defined as the cokernel

$$
C_{\mathrm{c}}^{\infty}(M) \stackrel{P_{M}}{\longrightarrow} C_{\mathrm{c}}^{\infty}(M) \longrightarrow \mathcal{V}(M):=C_{\mathrm{c}}^{\infty}(M) / P_{M}\left(C_{\mathrm{c}}^{\infty}(M)\right) .
$$

Because $C_{\mathrm{c}}^{\infty}:$ Loc $\rightarrow \mathbf{V e c}_{\mathbb{R}}$ is a cosheaf for (causally convex) open covers and $P: C_{\mathrm{c}}^{\infty} \rightarrow C_{\mathrm{c}}^{\infty}$ is a natural transformation, it follows that $\mathcal{V}: \mathbf{L o c} \rightarrow \mathbf{V e c}_{\mathbb{R}}$ is a cosheaf too. Consider the complexified symmetric algebra $\operatorname{Sym}_{\mathbb{C}}(\mathcal{V}(M)) \in \mathbf{C A l g}$, which is a commutative algebra in the closed symmetric monoidal category $\left(\operatorname{Vec}_{\mathbb{C}}, \otimes, \mathbb{C}, \tau\right.$ ) of complex vector spaces. This algebra is deformed to a noncommutative algebra by introducing a $\star$-product. For this we first define a (de Rham type) differential $\mathrm{d}$ : $\operatorname{Sym}_{\mathbb{C}}(\mathcal{V}(M)) \rightarrow \operatorname{Sym}_{\mathbb{C}}(\mathcal{V}(M)) \otimes \mathcal{V}(M)$ by setting on monomials

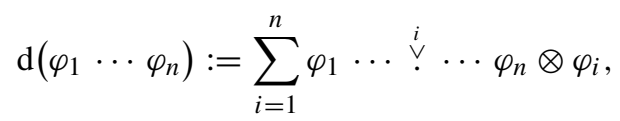




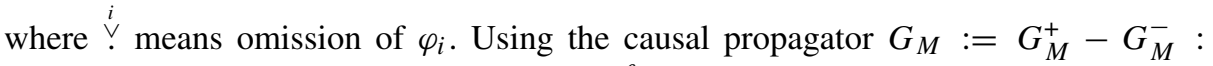
$\mathcal{V}(M) \rightarrow \operatorname{ker} P_{M}$ and the integration map $\int_{M}: \mathcal{V}(M) \otimes \operatorname{ker} P_{M} \rightarrow \mathbb{R}, \varphi \otimes \Phi \mapsto$ $\int_{M} \varphi \Phi \operatorname{vol}_{M}$, we define the bi-differential operator

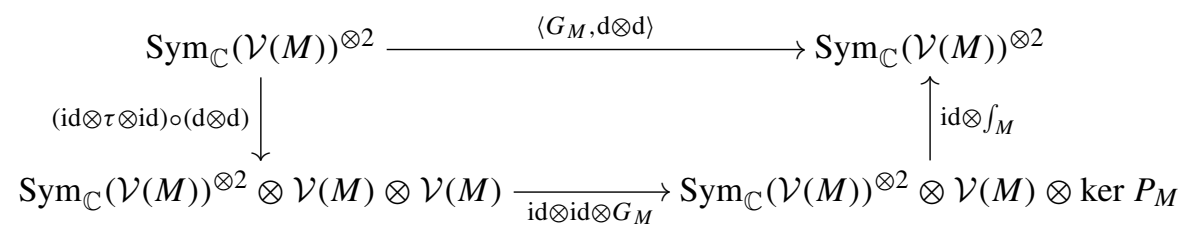

where we recall that $\tau$ is the symmetric braiding on $\mathbf{V e c}_{\mathbb{C}}$, i.e. the flip map. The $\star$-product $\star_{M}: \operatorname{Sym}_{\mathbb{C}}(\mathcal{V}(M))^{\otimes 2} \rightarrow \operatorname{Sym}_{\mathbb{C}}(\mathcal{V}(M))$ is then defined by composing

$$
\operatorname{Sym}_{\mathbb{C}}(\mathcal{V}(M))^{\otimes 2} \stackrel{\exp \left(\frac{i}{2}\left\langle G_{M}, \mathrm{~d} \otimes \mathrm{d}\right\rangle\right)}{\longrightarrow} \operatorname{Sym}_{\mathbb{C}}(\mathcal{V}(M))^{\otimes 2} \stackrel{\cdot M}{\longrightarrow} \operatorname{Sym}_{\mathbb{C}}(\mathcal{V}(M))
$$

where $\cdot M$ denotes the commutative product on $\operatorname{Sym}_{\mathbb{C}}(\mathcal{V}(M))$. (The exponential series converges because it terminates for polynomials.) Setting $\mathfrak{A}_{\mathrm{KG}}(M):=\left(\operatorname{Sym}_{\mathbb{C}}(\mathcal{V}(M))\right.$, $\left.\star_{M}, \eta_{M}\right) \in$ Alg with $\eta_{M}$ the unit of $\operatorname{Sym}_{\mathbb{C}}(\mathcal{V}(M))$, for all $M \in \mathbf{L o c}$, defines a Cauchy constant additive algebraic quantum field theory $\mathfrak{A}_{\mathrm{KG}} \in \mathbf{A Q F T}{ }^{\text {add }, c}$. Note that additivity is a consequence of $\mathcal{V}:$ Loc $\rightarrow \mathbf{V e c}_{\mathbb{R}}$ being a cosheaf.

Theorem 5.1 provides a corresponding Cauchy constant additive time-orderable prefactorization algebra $\mathfrak{F}_{\mathrm{KG}}:=\mathbb{F}\left[\mathfrak{A}_{\mathrm{KG}}\right] \in$ tPFA $^{\text {add, } c}$. To get some intuition on what this prefactorization algebra does, let us analyze the explicit form of the binary timeordered products $\mathfrak{F}_{\mathrm{KG}}(f): \mathfrak{F}_{\mathrm{KG}}\left(M_{1}\right) \otimes \mathfrak{F}_{\mathrm{KG}}\left(M_{2}\right) \rightarrow \mathfrak{F}_{\mathrm{KG}}(N)$. In the case where $\underline{f}=\left(f_{1}, f_{2}\right): \underline{M} \rightarrow \bar{N}$ is time-ordered, i.e. $J_{N}^{+}\left(f_{1}\left(M_{1}\right)\right) \cap f_{2}\left(M_{2}\right)=\emptyset$, we obtain from (4.7), (5.12) and the support properties of $G_{N}^{ \pm}$that

$$
\mathfrak{F}_{\mathrm{KG}}(\underline{f})=\cdot_{N} \circ \exp \left(\frac{i}{2}\left\langle G_{N}^{+}, \mathrm{d} \otimes \mathrm{d}\right\rangle\right) \circ\left(\mathfrak{A}_{\mathrm{KG}}\left(f_{1}\right) \otimes \mathfrak{A}_{\mathrm{KG}}\left(f_{2}\right)\right) \quad(\underline{f} \text { time-ordered }) .
$$

In the case where $\underline{f}=\left(f_{1}, f_{2}\right): \underline{M} \rightarrow N$ is anti-time-ordered, i.e. $J_{N}^{+}\left(f_{2}\left(M_{2}\right)\right) \cap$ $f_{1}\left(M_{1}\right)=\emptyset$, we obtain

$\mathfrak{F}_{\mathrm{KG}}(\underline{f})=\cdot_{N} \circ \exp \left(\frac{i}{2}\left\langle G_{N}^{-}, \mathrm{d} \otimes \mathrm{d}\right\rangle\right) \circ\left(\mathfrak{A}_{\mathrm{KG}}\left(f_{1}\right) \otimes \mathfrak{A}_{\mathrm{KG}}\left(f_{2}\right)\right) \quad(\underline{f}$ anti-time-ordered $)$.

Using again the support properties of $G_{N}^{ \pm}$, we observe that the the two cases in (5.13) can be combined into a single formula

$$
\mathfrak{F}_{\mathrm{KG}}(\underline{f})=\cdot_{N} \circ \exp \left(i\left\langle G_{N}^{\mathrm{D}}, \mathrm{d} \otimes \mathrm{d}\right\rangle\right) \circ\left(\mathfrak{A}_{\mathrm{KG}}\left(f_{1}\right) \otimes \mathfrak{A}_{\mathrm{KG}}\left(f_{2}\right)\right) \quad(\underline{f} \text { time-orderable }),
$$

where $G_{N}^{\mathrm{D}}:=\frac{1}{2}\left(G_{N}^{+}+G_{N}^{-}\right)$is the so-called Dirac propagator, that is valid for every time-orderable tuple $\left(f_{1}, f_{2}\right)$. In perturbative algebraic quantum field theory (see e.g. $\left[\right.$ FR13, Rej16]), the products $\cdot_{\mathcal{T}_{N}}:=\cdot_{N} \circ \exp \left(i\left\langle G_{N}^{\mathrm{D}}, \mathrm{d} \otimes \mathrm{d}\right\rangle\right)$ are called time-ordered products. 
Our observations in this subsection can thus be summarized as follows: The prefactorization algebra $\mathfrak{F}_{\mathrm{KG}} \in \mathbf{t P F A}^{\text {add, } c}$ corresponding to the free Klein-Gordon theory $\mathfrak{A}_{\mathrm{KG}} \in \mathbf{A Q F T}{ }^{\text {add, } c}$ encodes the usual time-ordered products obtained by the Dirac propagator. This agrees with the observations in [GR17].

Acknowledgements. We would like to thank the anonymous referees for useful comments that helped us to improve this manuscript. The work of M.B. is supported by a research grant funded by the Deutsche Forschungsgemeinschaft (DFG, Germany) (Grant No. 367130593). M.P. is supported by a PhD scholarship of the Royal Society (UK). A.S. gratefully acknowledges the financial support of the Royal Society (UK) (Grant Nos. UF150099, RG160517, RGF $\backslash E A \backslash 180270$ ) through a Royal Society University Research Fellowship, a Research Grant and an Enhancement Award.

Open Access This article is distributed under the terms of the Creative Commons Attribution 4.0 International License (http://creativecommons.org/licenses/by/4.0/), which permits unrestricted use, distribution, and reproduction in any medium, provided you give appropriate credit to the original author(s) and the source, provide a link to the Creative Commons license, and indicate if changes were made.

Publisher's Note Springer Nature remains neutral with regard to jurisdictional claims in published maps and institutional affiliations.

\section{References}

[BGP07] Bär, C., Ginoux, N., Pfäffle, F.: Wave equations on Lorentzian manifolds and quantization. Eur. Math. Soc. Zürich (2007). arXiv:0806.1036 [math.DG]

[BS17] Benini, M., Schenkel, A.: Quantum field theories on categories fibered in groupoids. Commun. Math. Phys. 356, 19 (2017). arXiv:1610.06071 [math-ph]

[BS19] Benini, M., Schenkel, A.: Higher structures in algebraic quantum field theory. Fortschr. Phys. arXiv:1903.02878 [hep-th] (to appear)

[BSS15] Benini, M., Schenkel, A., Szabo, R.J.: Homotopy colimits and global observables in Abelian gauge theory. Lett. Math. Phys. 105, 1193 (2015). arXiv:1503.08839 [math-ph]

[BSW17] Benini, M., Schenkel, A., Woike, L.: Operads for algebraic quantum field theory. arXiv:1709.08657 [math-ph]

[BSW19a] Benini, M., Schenkel, A., Woike, L.: Involutive categories, colored $*$-operads and quantum field theory. Theory Appl. Categ. 34, 13 (2019). arXiv:1802.09555 [math.CT]

[BSW19b] Benini, M., Schenkel, A., Woike, L.: Homotopy theory of algebraic quantum field theories. Lett. Math. Phys. 109(7), 1487 (2019). arXiv:1805.08795 [math-ph]

[BS06] Bernal, A.N., Sanchez, M.: Further results on the smoothability of Cauchy hypersurfaces and Cauchy time functions. Lett. Math. Phys. 77, 183 (2006). arXiv:gr-qc/0512095

[BDFY15] Brunetti, R., Dappiaggi, C., Fredenhagen, K., Yngvason, J.: Advances in Algebraic Quantum Field Theory. Springer, Heidelberg (2015)

[BFV03] Brunetti, R., Fredenhagen, K., Verch, R.: The generally covariant locality principle: a new paradigm for local quantum field theory. Commun. Math. Phys. 237, 31 (2003). arXiv:math-ph/0112041

[CG17] Costello, K., Gwilliam, O.: Factorization Algebras in Quantum Field Theory. Volume 1, New Mathematical Monographs 31. Cambridge University Press, Cambridge (2017)

[Few13] Fewster, C.J.: Endomorphisms and automorphisms of locally covariant quantum field theories. Rev. Math. Phys. 25, 1350008 (2013). arXiv:1201.3295 [math-ph]

[FV12] Fewster, C.J., Verch, R.: Dynamical locality and covariance: what makes a physical theory the same in all spacetimes? Ann. Henri Poincaré 13, 1613 (2012). arXiv:1106.4785 [math-ph]

[FR13] Fredenhagen, K., Rejzner, K.: Batalin-Vilkovisky formalism in perturbative algebraic quantum field theory. Commun. Math. Phys. 317, 697 (2013). arXiv:1110.5232 [math-ph]

[Fre17] Fresse, B.: Homotopy of Operads and Grothendieck-Teichmüller Groups. Part 1: The Algebraic Theory and Its Topological Background, Mathematical Surveys and Monographs 217. American Mathematical Society, Providence (2017)

[GR17] Gwilliam, O., Rejzner, K.: Relating nets and factorization algebras of observables: free field theories. arXiv:1711.06674 [math-ph]

[ONe83] O'Neill, B.: Semi-Riemannian Geometry. Academic Press, New York (1983) 
[Rej16] Rejzner, K.: Perturbative Algebraic Quantum Field Theory: An Introduction for Mathematicians. Mathematical Physics Studies. Springer, Cham (2016)

[Yau16] Yau, D.: Colored Operads. Graduate Studies in Mathematics 170. American Mathematical Society, Providence (2016)

Communicated by Y. Kawahigashi 